Materials and Structures manuscript No.

(will be inserted by the editor)

\title{
Review: Optical Fiber Sensors for Civil Engineering
}

\section{Applications}

\author{
Christopher K.Y. Leung · Kai Tai Wan · Daniele
}

Inaudi · Xiaoyi Bao · Wolfgang Habel · Zhi

Zhou · Jinping Ou · Masoud Ghandehari · Hwai

Chung Wu · Michio Imai

Received: date / Accepted: date

Christopher K.Y. Leung

Hong Kong University of Science and Technology, Hong Kong

E-mail: ckleung @ust.hk

Kai Tai Wan

Nano and Advanced Materials Institute Limited, Hong Kong

E-mail: ktwan@nami.org.hk

Daniele Inaudi

Smartec, Switzerland

Xiaoyi Bao

University of Ottwa, Canada

Wolfgang Habel

BAM, Germany

Zhi Zhou

Harbin institute of Technology, China

Jinping $\mathrm{Ou}$

Dalian University of Technology, China 
Masoud Ghandehari

Polytechnic Institute of New York University, USA

Hwai Chung Wu

Wayne State University, USA

Michio Imai

Kajima Technical Research Institute, Japan

This work is derived from a study by the RILEM Technical Committee on Optical Fiber Sensors for Civil Engineering Applications. Other members of the committee are:

Farhad Ansari

University of Illinois, Chicago, USA

Evangelos Astrinidis

CRD Group, Greece

Muhammed Basheer

Queen's University, Belfast, UK

Rolf Broennimann

EMPA, Switzerland

Paulo Cruz

University of Minho, Portugal

Wei-Liang Jin

Zhejiang University, China

Ki Soo Kim

Stijn Matthys

Ghent University, Belgium

Aftab Mufti

University of Manitoba

John Newhook 
Abstract Optical fiber sensor (OFS) technologies have developed rapidly over the last few decades, and various types of OFS have found practical applications in the field of civil engineering. In this paper, which is resulting from the work of the RILEM technical committee "Optical fiber sensors for civil engineering applications", different kinds of sensing techniques, including change of light intensity, interferometry, fiber Bragg grating, adsorption measurement and distributed sensing, are briefly reviewed to introduce the basic sensing principles. Then, the applications of OFS in highway structures, building structures, geotechnical structures, pipelines as well as cables monitoring are described, with focus on sensor design, installation technique and sensor performance. It is believed that the State-ofthe-Art review is helpful to engineers considering the use of OFS in their projects, and can facilitate the wider application of OFS technologies in construction industry.

Keywords optical fiber sensors · monitoring · fiber Bragg grating · distributed sensor · interferometry $\cdot$ time domain reflectometry $\cdot$ frequency domain reflectometry

Dalhousie University, Canada

Stephanie Schuler

BAM, Germany

Joe Seinfield

Purdue University, USA

Gamil Tadros

Speco Engineering, Ltd, Canada

Leon Wegner

University of Saskatchewan, Canada

Zhishen Wu

Ibaraki University, Japan 


\section{Introduction}

Infrastructure decay is a big problem faced by many developed countries in the world. After years of service, many structures, such as bridges, tunnels, dams and power plants, are showing severe deterioration. To ensure safety of these structures and to perform proper maintenance/rehabilitation, there is a need to monitor the structural condition or "health" over time. Also, for important new structures, sensors are often installed during the construction phase so minor degradations can be identified at an early stage. Low-cost minor repair can then be performed to avoid major damages that require costly renovations or even reconstruction. The use of new materials in construction also creates a need for monitoring. For example, fiber reinforced polymers (FRP) are now being employed for the fabrication of components for bridge construction and repair. While the performance of these materials has been verified in the laboratory, real-world experience is still limited. The installation of monitoring system in a FRP structure allows engineers to assess its in-situ performance and ensure safe operation. The obtained information can also be employed to improve the future design of structures with these new materials.

The use of any structural health monitoring system, if correctly designed and used, can have a positive impact on the lifetime cost of a structure. By providing quantitative information on the state of a structure, it allows a better management of its maintenance and inspections, resulting in a positive benefit to cost comparison. Conventionally, electrical gauges and transducers have been the technology of choice for measuring many physical and chemical parameters. Recently, fiber optic based monitoring systems have been successfully developed and employed. In optical sensing, the sensing element is part of an optical fiber which transforms a change in the monitored parameter into a corresponding change in the properties of the guided light, which can be its intensity, phase, spectral content, polarization 
state or a combination of these. The sensor is linked to the data acquisition system through an optical fiber communication link. In some cases, the signal from many sensors can be transmitted through the same fiber link. Field installation can hence be simplified.

For various kinds of optical fiber sensors, there are corresponding data acquisition units that would convert the optical measurement into the parameter of interest. Being a new technology, an optical fiber sensing system is more expensive than its electrical counterpart, with the exception of distributed sensing where the cost per measurement point can be much lower than individual conventional sensors. However, optical sensing technology possesses a number of advantages that justify its use in practical applications. Optical fiber sensors (OFS) are intrinsically immune to electromagnetic interference (EMI), such as those generated by lightning strikes, nearby power lines or wireless communication systems. By design, OFS are intrinsically safe and naturally explosion proof, making them particularly suitable for health monitoring applications of risky civil structures such as gas pipelines or chemical plants. Another advantage of OFS is their small size. The diameter of bare OFS, usually in the range of $125 \mu \mathrm{m}$ to $1000 \mu \mathrm{m}$, makes them applicable in space-restricted environments such as thin composite structures. The ability of measuring over long distances (of several tens of kilometers), without the need for any electrically active component, is an important feature in the monitoring of large and remote structures such as pipelines or multiple bridges along a single highway. Depending on the length of the sensing element, the sensor can be configured into a "point" sensor performing measurement at a particular point or an "integrated" (or long-gauge) sensor obtaining the averaged information over a particular region. Along a single fiber, several "point" sensors can be multiplexed to obtain information at a number of specific points. Also, sensors on the same fiber can be configured to measure different parameters (e.g., strain and temperature). More importantly, since an optical fiber possesses sensing capacity at any point along its length, it is possible to perform truly "distributed" 
measurement along the fiber. The capability for multiplexing and distributed sensing extends the repertoire of existing monitoring techniques. If designed and packaged properly, OFS can be very robust, and last for a long time in the most challenging environments. With the longterm durability and low maintenance of OFS, it is possible for the life-cycle cost of an optical sensing system to be lower than an electrical one. With the above advantages, OFS have been employed (though in limited extent) to replace conventional sensors in structural monitoring applications. They have also been used in experimental research as tools to provide accurate and stable measurements in the laboratory. Actually, by exploiting the special properties of optical fibers, novel sensors can be designed to provide sensing capabilities not achievable with existing instruments, and hence make possible the acquisition of new information useful to engineers.

As discussed above, optical fiber sensing systems can replace existing electrical systems if (i) conventional sensor fails to satisfy performance requirements (e.g., stability, accuracy, durability, etc) under specific conditions, or (ii) the optical sensor can provide useful information not obtainable with conventional sensors. In many cases it is also possible to combine traditional sensors and fiber optic sensors (e.g. vibrating-wire piezometers and fiber optic distributed leakage detection systems in the same dam), to get the best of both worlds. Data from the two systems can be combined at the processing stage. To identify suitable applications of the optical fiber sensor, a good understanding of the principles and advantages of such sensors is required. Such understanding, however, is often lacking for civil engineers. The major objective of this paper is to review the current State-of-the-Art for the application of optical fiber sensors in civil engineering. Such information can hopefully facilitate the further application of this new technology in civil engineering practice. In the next section, common optical sensing principles are first briefly described. Then, practical applications of 
OFS in monitoring highway structures, building structures, geotechnical structures, pipelines and cables will be discussed in Section 3 .

\section{Optical Fiber Sensing Principles}

In this section, common optical fiber sensing principles are reviewed to provide the required background for readers to appreciate the various practical applications to be described in Section 3. To serve this purpose, the description for each kind of sensor will be kept very brief, in order not to duplicate the effort of other works in the literature. In general every fiber optic sensor is designed to somehow change the properties of the light that is transmitted through or reflected from the sensors. Changes can include variations of intensity, polarization and spectral content of phase. Readers interested in more information, should refer to the cited references.

\subsection{Change of light intensity}

The intensity of light signal passing through an optical fiber or reflected from its end is easy to measure. The breakage of a fiber can result in a sudden drop in the forward signal (or a sudden increase in the reflected signal). If a fiber is bonded strongly to a structure, a crack formed in the structure can induce fiber breakage [?,?]. If several parallel fibers are placed perpendicular to the anticipated direction of crack growth, the propagation of a crack can then be monitored [?]. The major disadvantage of these sensors lies in the difficulty in controlling the failure consistency. To achieve consistency, all fibers need to be coupled to the structure in exactly the same way - a task not easily accomplished on site. Also, since glass is a brittle material with high material variability, even fibers bond in the same way may fail under very different strains. Signal intensity change due to fiber breakage is hence not very useful if 
accurate quantitative information is required. Bending of an optical fiber is another way to induce change in the light intensity. In a straight optical fiber, the light wave is guided within the core of the optical fiber. As the fiber is bent, part of the light will escape through the cladding into the surrounding. The light intensity passing through the fiber is hence reduced. Sensors based on a fiber loop have been developed by Wolff and Messelier [?] and Ansari et al [?]. For these sensors, the fiber loops are coupled to a concrete structure in such a way that cracking will induce change in the loop geometry and hence the amount of bending loss. The formation and opening of cracks can hence be monitored.

Monitoring of signal intensity can also be employed to study the chemical condition of a certain environment. For example, a thin reflective film can be coated on the smooth cleaved end of an optical fiber. If the presence of a certain chemical will lead to depletion of the film, the fiber can be employed as a sensor for that particular chemical. By leaving the fiber in a certain environment, a sudden drop in reflected signal indicates significant loss of the coated film, which reveals the presence of the chemical.

Intensity monitoring exhibits an inherent problem. The light source itself may fluctuate in power over time. Also, local microbending along the connecting fiber induces losses that vary with time and the insertion loss may vary if the connection is removed after each measurement. Therefore, the measured change in signal intensity is not necessarily due to change in the measured parameter alone. If the magnitude of a reflected signal is to be measured, the above-mentioned problem can be alleviated with the use of a time domain reflectometer (which will be discussed further in Section 2.5), where the reflected signal is compared directly to the signal right before the reflection point to obtain a relative reading that is not affected by light intensity of the source or losses at other points. 


\subsection{Interferometry}

Interferometry involves the combination of two separate light signals from the same source. To explain the technique, it is best to consider the Mach-Zehnder interferometer in which a light beam is split and coupled into two separate fibers. One fiber is attached to a structure while the other is left free. At the other end, signal from the two fibers are re-combined and monitored. When the structure is loaded, the length of the attached fiber will change, leading to an increase in optical path for the light. The corresponding phase shift will lead to a periodic variation of light intensity when light from the two fibers are re-combined. Since each fringe (or complete periodic variation of light intensity) corresponds to a change in length equal to the wavelength of light, which is around one micron, counting the number of fringes can provide enough resolution for the measurement of relative large strains. For small strain measurements, feedback loops can be employed to convert the periodic intensity into a linearly varying in voltage output [?].

The Mach-Zehnder interferometer has several disadvantages. Two fibers need to be used at each sensing point. Very tight control of bonded fiber length is required to ensure a consistent gauge length, so the measured length change can be correctly translated into a strain value. Also, when low-cost light sources such as LED are employed, the low coherence length requires close matching of the length of the attached and free fibers. In the instrumentation of large structures, long fiber lengths are required and close length matching can be very difficult.

To overcome the above shortcomings, the extrinsic Fabry-Perot Interferometer (EFPI) can be employed $[?, ?, ?]$. The EFPI is constituted by a capillary tube containing two cleaved optical fibers facing each other (Fig. 1), but leaving an air gap of a few microns or tens of microns between them. When light is launched into one of the fibers, a back-reflected 


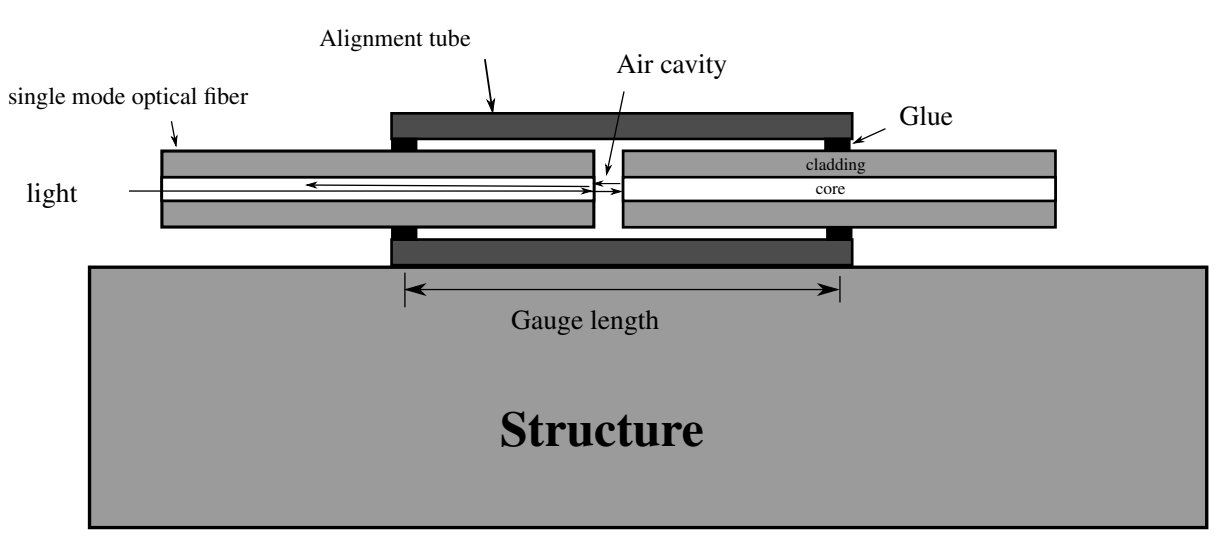

Fig. 1 Functional principle of extrinsic Fabry-Perot interferometer

interference signal is obtained from the reflections of the incoming light on the glass-to-air and air-to-glass interfaces, respectively. This interference can be demodulated using coherent or low-coherence techniques to reconstruct the changes in the fiber spacing. Since the two fibers are attached to the capillary tube near its two extremities (with a typical spacing of $10 \mathrm{~mm}$ ), the gap change will correspond to the average strain variation between the two attachment points.

Using the principle of white light interferometry, researchers at the Swiss Federal Institute of Technology in Lausanne (EPFL) have developed the SOFO system, which is a long-gauge fiber optic deformation sensor with a resolution in the micrometer range $[?, ?, ?, ?]$. The sensor is now commercialized by SMARTEC and Roctest Group. The functional principle of the SOFO system is shown schematically in Fig. 2.

The sensor consists of a pair of single-mode fibers placed inside a tube which can be attached to the structure to be monitored. One of the fibers, called measurement fiber, is in mechanical contact with the host structure, being attached to it at its two extremities and pre-stressed in-between (see Fig. 2), while the other, the reference fiber, is placed loose in the same tube. Deformation of the structure will then result in a change of the length 


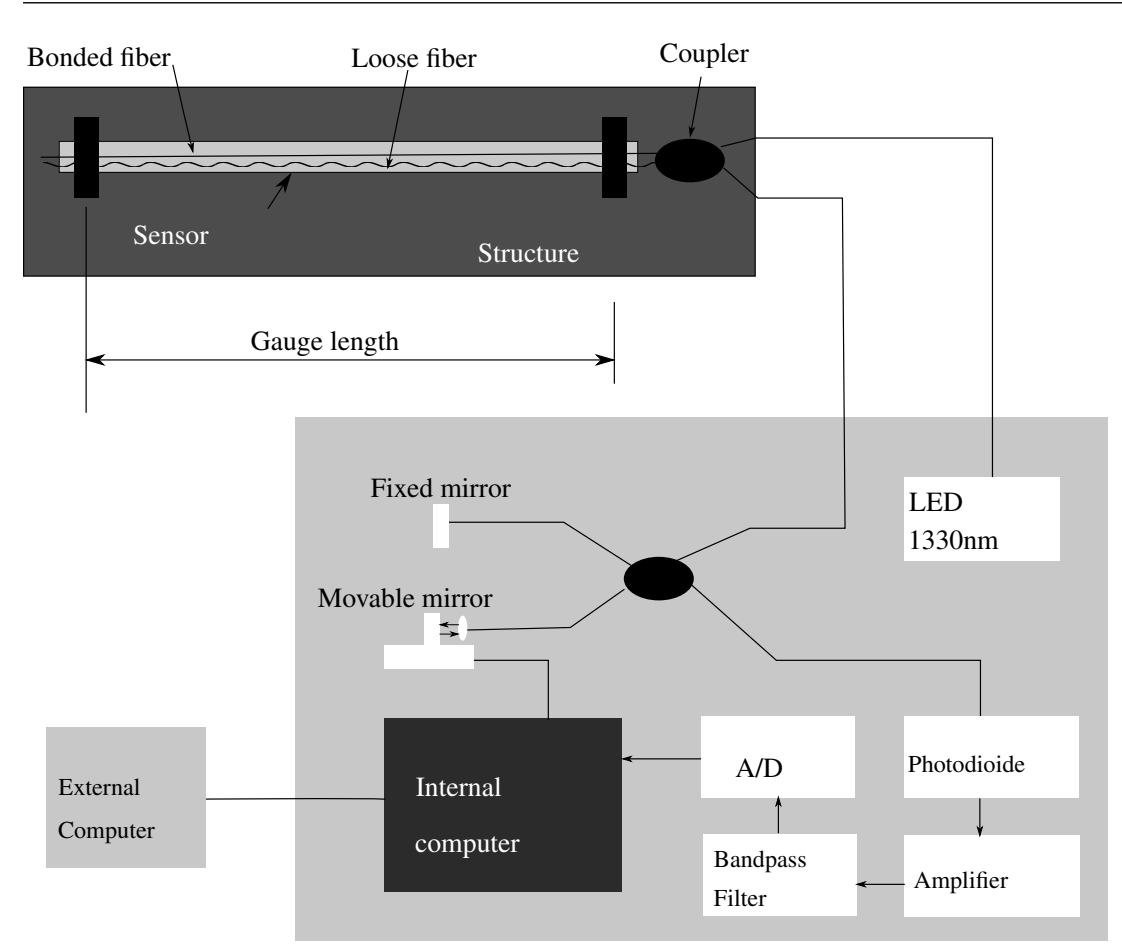

\section{SOFO Readout System}

Fig. 2 Functional principle of SOFO system

difference between these two fibers. To make an absolute measurement of this path unbalance, a low-coherence double Michelson interferometer is used. The first interferometer is made of the measurement and reference fibers, while the second is contained in a portable reading unit. This second interferometer can introduce, by means of a scanning mirror, a known path unbalance between its two arms. Because of the reduced coherence of the source used (the 1.3 micron radiation of a LED), interference fringes are detectable only when the reading interferometer compensates exactly the length difference between the fibers in the structure. With the unbalance of the reading interferometer determined by the position of the scanning mirror, the strain on the SOFO sensor can be easily obtained. Note that this sensor provides absolute strain reading, not affected by light source intensity or losses along the optical link 
as well as change of temperature that affects both fibers equally so the effects cancel each other.

\subsection{Fiber Bragg Gratings}

A Bragg grating is a periodic variation of refractive index along the length of the fiber, normally about $10 \mathrm{~mm}$ in total length. Gratings with periods $\Lambda$ are commonly fabricated by holographic [?] or phase-mask technique [?,?]. The working principle of the Bragg grating sensor is shown in Fig. 3. When a broadband or tunable signal passes through the optical fiber, the wavelength corresponding to the period of index variation $\left(\lambda_{B}\right)$ will be preferentially reflected. The relationship between Bragg wavelength and the periodic spacing of the grating is given by Equation $1[?, ?]$.

$$
\lambda_{\mathrm{B}}=2 n_{\mathrm{e}} \Lambda
$$

where $n_{\mathrm{e}}$ is the effective refractive index of the core of the grating. When the fiber is under mechanical strain $\left(\varepsilon_{\text {mech }}\right)$ or temperature variation $(\Delta T)$, the index variation period will change, leading to a peak shift in the reflected wavelength. To perform measurement, a tunable laser, spectrometer or wavelength filter is normally employed. The relationship between the shift of Bragg wavelength and the total induced axial strain is given by Equation 2 .

$$
\frac{\Delta \lambda_{B}}{\lambda_{B}}=\left(1-p_{e}\right) \varepsilon_{\mathrm{mech}}+(\alpha+\zeta) \Delta T
$$

where $p_{e}, \alpha$ and $\zeta$ are strain-optic coefficient, thermal expansion coefficient and thermaloptic coefficient, respectively. To compensate the thermal induced strain, additional thermal compensation FBG sensor should be installed in the same sensing region. The wavelength of the thermal compensation FBG sensor $\left(\lambda_{T}\right)$ is different from the mechanical strain sensitive FBG sensor and it should be isolated from any mechanical strain. The mechanical induced 


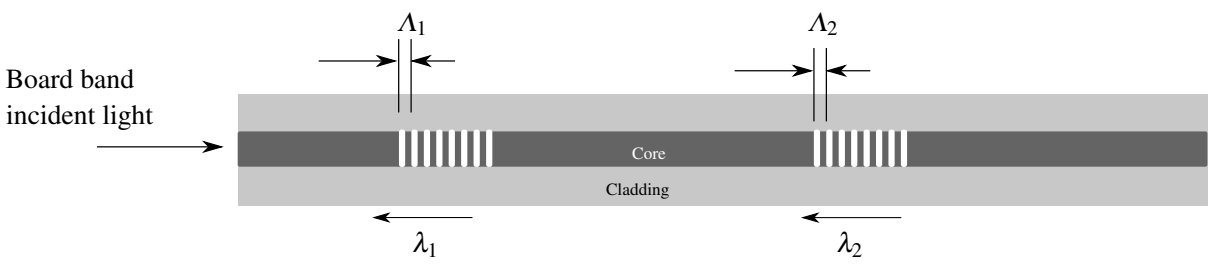

Fig. 3 Working principle of fiber Bragg grating

strain can be estimated from the change in Bragg wavelength of the total strain and thermal induced strain by Equation 3 .

$$
\varepsilon_{\mathrm{mech}}=\frac{1}{1-p_{e}}\left(\frac{\Delta \lambda_{B}}{\lambda_{B}}-\frac{\Delta \lambda_{T}}{\lambda_{T}}\right)
$$

When strain values are required at more than one point along the fiber, gratings of different periods can be placed at the corresponding points. The reflected signal will then exhibit a number of peaks corresponding to individual gratings. By monitoring the shifts of all the peaks, strain at each point can be deduced. Typically, 4 to 16 gratings can be measured on a single fiber line. It should be noticed that since the gratings have to share the spectrum of the source used to illuminate them, there is a trade-off between the number of gratings and the dynamic range of their measurements.

\subsection{Absorption measurement}

Absorption measurement is normally employed for the sensing of chemicals. For example, when light is sent through an optical fiber into a chemical environment with preferential absorption of a particular wavelength, the spectrum of the reflected signal will show a drop in the absorbed wavelength. Similarly, one can design a sensor with two flat-end fibers separated by a small gap. Light passing through the gap will exhibit a change in its spectrum if chemicals that absorb certain wavelengths are present in the surrounding environment and enter the inter-fiber gap. Absorption spectroscopy is to measure the electromagnetic radiation 
absorbed by atoms, molecules or other chemical entities. In microwave spectroscopy, the absorption is accompanied by a transition from one rotational energy level of a molecule to another. In infrared spectroscopy, the transition is from one vibrational energy level of a molecule to another. In ultraviolet-visible electronic absorption spectroscopy, transition levels relate to valence electrons in the molecule.

For a step-index multimode optical fiber, the number of possible modes guided through the fiber increases with increasing core diameter and wavelength. The energy guided through the core by total internal reflection also generates a surface-specific, electromagnetic disturbance at the interface between the two dielectric media (the core and the cladding). There is a standing wave at every point of interface incidence. This harmonic wave, which penetrates the cladding over a small distance, is called the evanescent field. For chemical sensing, the cladding is replaced by chromoionophores which are indicator molecules that, in the presence of specific ions $\left(\mathrm{H}^{+}, \mathrm{Ca}^{2+}, \mathrm{Cl}^{-}, \mathrm{K}^{+}\right.$, etc. $)$, exhibit a change in color, i.e. a change in the absorption spectrum. As chromoionophores exhibiting unique absorption spectra with different wavelengths of maximum absorption $\left(\lambda_{\max }\right)$ in the absence/presence of the specific ions, chemical sensing can be performed by sending a broad band light signal into the fiber and measuring the spectrum at the other side.

2.5 Time domain reflectometry

Developed for telecommunication applications, OTDRs (Optical Time Domain Reflectometers) have been the starting point of distributed sensing techniques. Conventionally, the Rayleigh scattered light has been used to measure the attenuation profiles of long-haul fiber optics links. In OTDR technique, a short optical pulse is launched into the fiber and a photo detector measures the amount of light that is backscattered as the pulse propagates down the 


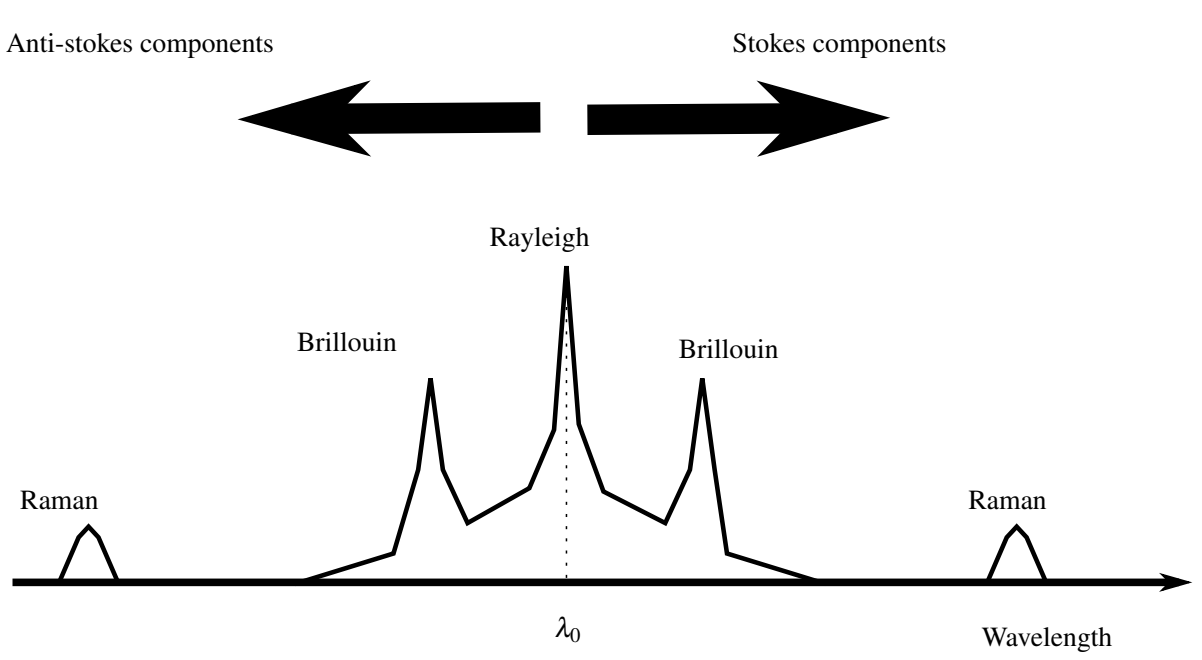

Fig. 4 Optical scattering components in optical fibers

fiber. The detected signal, the so-called Rayleigh signature, presents an exponential decay with time that is directly related to the linear attenuation of the fiber. The time information is converted to distance information if the speed of light is known, similar to radar detection techniques. In addition to the information on fiber losses, the OTDR profiles are very useful to localize breaks, to evaluate splices and connectors, and in general to assess the overall quality of a fiber link.

Raman and Brillouin scattering phenomena have been used for distributed sensing applications over the past few years. Raman was first proposed for sensing applications in the $80 \mathrm{~s}$, whereas Brillouin was introduced later as a way to enhanced the range of OTDR and then for strain and/or temperature monitoring applications. Fig. 4 shows schematically the spectrum of the scattered light from a single wavelength $\lambda_{o}$ in an optical fiber. Note that the Rayleigh scattering has frequency components close to the forward-propagating light. However, both Raman and Brillouin scattering effects are associated with different dynamic non-homogeneities in the silica and therefore have completely different spectral characteristics. 
The Raman scattered light is caused by thermally influenced molecular vibrations. Consequently, the backscattered light carries the information on the local temperature where the scattering occurred $[?, ?]$. The amplitude of the anti-stokes component is strongly temperature dependent whereas the amplitude of the stokes component is not. Measuring the intensity ratio between these bands, called the stokes and anti-stokes emissions, it is possible to calculate the temperature at any given point along the fiber line [?]. Typical spatial resolutions for distributed temperature measurements are of the order of one meter. Also, the temperature resolution is $0.2^{\circ} \mathrm{C}$.

Since the magnitude of the spontaneous Raman backscattered light is quite low, high numerical aperture multimode fibers are used in order to maximize the guided intensity of the backscattered light. However, the relatively high attenuation characteristics of multimode fibers limit the distance range of Raman-based systems to approximately $30 \mathrm{~km}$.

Brillouin scattering $[?, ?, ?, ?]$ occurs due to the interaction between the propagating optical signal and thermally excited acoustic waves in the $\mathrm{GHz}$ range present in the silica fiber, giving rise to frequency shifted components. It can be seen as the diffraction of light on a dynamic grating generated by an acoustic wave (an acoustic wave is actually a pressure wave which introduces a modulation of the index of refraction through the elasto-optic effect). The diffracted light experiences a Doppler shift since the grating propagates at the acoustic velocity in the fiber. The acoustic velocity is directly related to the density of the medium that is temperature and strain dependent. As a result, the so-called Brillouin frequency shift carries the information about the local temperature and strain of the fiber.

The active stimulation of Brillouin scattering can be achieved by using 2 optical light waves. In addition to the optical pulse usually called the pump, a continuous wave (CW) optical signal, the so-called probe signal is used to probe the Brillouin frequency profile of the fiber [?,?]. A stimulation of the Brillouin scattering process occurs when the frequency 
difference (or wavelength separation) of the pulse and the CW signal corresponds to the Brillouin shift (resonance condition) and provided that both optical signals are counterpropagating in the fiber. The interaction leads to a larger scattering efficiency resulting in an energy transfer from the pulse to the probe signal, and an amplification of the probe signal. The frequency difference between pulse and probe can be scanned for precise and global mapping of the Brillouin shift along the sensing fiber. At every location, the maximum of the Brillouin gain is computed and the information transformed to temperature or strain using the appropriate calibration coefficients. The probe signal intensity can be adjusted to acceptable levels for low-noise fast acquisition whatever the measurement conditions and fiber layout, thus solving the main problem that is generally associated with distributed sensing based on spontaneous light scattering.

For applications where the sensing area is located more than $25 \mathrm{~km}$ away from the instrument, it is possible to boost the optical signal using light amplifiers: the so-called range extender modules. The module performs active signal regeneration by using optical amplification techniques similar to those extensively used in optical telecommunications. The modules can be cascaded leading to remote distances in excess of hundreds of kilometers.

For distributed temperature measurements, both the Brillouin and Raman OTDR can be employed. For distributed strain sensing, the Brillouin OTDR is the most powerful. Since the Brillouin frequency shift depends on both the local strain and temperature of the fiber, the sensor configuration will determine the actual sensitivity of the system. For measuring temperature, it is sufficient to use a standard telecommunication cable that shields the optical fibers from mechanical strain. The fiber will therefore remain in its unstrained state and the frequency shifts can be unambiguously assigned to temperature variations. For strain measurement, the sensor configuration should be designed to ensure proper mechanical coupling between the sensor and the host structure along the whole length of the fiber. To 
resolve the cross-sensitivity to temperature variations, it is also necessary to install a reference fiber along the strain sensor. Knowing the frequency shift of the unstrained fiber allows an absolute strain measurement.

2.6 Frequency domain reflectometry

When the spatial resolution of OTDR systems is reduced to millimeters scale, it requires a data acquisition device with a bandwidth of $10 \mathrm{GHz}$ and a sampling rate of tens of G Sample/s. At the same time, the pulse generator should output sufficiently high energy in a very short duration and the light detection device should be sensitive enough to measure the scattered signal with sufficient signal-to-noise ratio. All these factors make the high spatial resolution OTDR-based distributed sensor very expensive. A cost effective distributed sensor with millimeter scale spatial resolution has been developed based on the optical frequency domain reflectometry (OFDR) of Rayleigh scattering [?,?,?,?]. Instead of measuring the intensity of Rayleigh backscattered signal, OFDR measures the interference fringes of the Rayleigh scattered light from a tunable laser source and a static reference fiber in frequency domain. The amplitude and phase in frequency domain is converted into time/spatial domain by inverse Fourier transform. Although Rayleigh scattering itself is independent of temperature and strain, a change of path difference between the sensing fiber and the reference fiber with fixed length of local oscillator in the interferometer provides temperature and strain variation induced phase differences through index variation, which can be measured at each fiber location (Fig. 5). The spatial resolution of $\operatorname{OFDR}\left(\Delta_{z}\right)$ is determined by the optical frequency sweep range of the tunable laser source $(\Delta F)$ as in Equation 4.

$$
\Delta_{z}=\frac{c}{2 n_{g} \Delta F}
$$




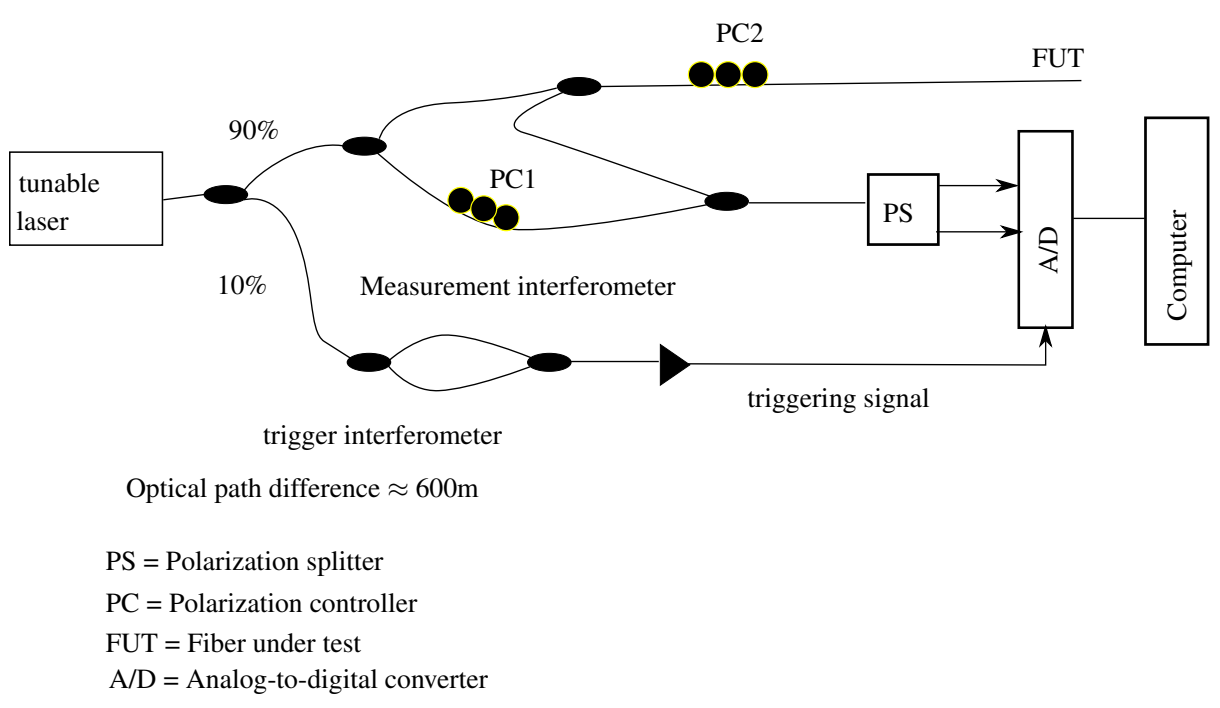

Fig. 5 Schematic diagram of optical frequency domain reflectometey

where $c$ and $n_{g}$ are the speed of light in vacuum and the group refractive index, respectively. In general, OFDR is suitable for short sensing length $(<100 \mathrm{~m})$ with no sharp change in strain and temperature between two measurements. By using polarization maintaining fiber, both temperature and strain can be obtained simultaneously.

The classical approach for measuring stimulated Brillouin scattering (SBS) in time domain is limited by similar constraints in light source, light detection components and data acquisition units as OTDR. To overcome the drawbacks, Brillouin optical frequency domain analysis (BOFDA) has been developed [?,?]. The spatial resolution of BOFDA system is determined by the modulation bandwidth of the pump beam $\Delta F$ as in Equation 4 . The amplitude of the pump beam is sinusoidally modulated. Fig. 6 shows the schematic diagram of a typical BOFDA system. The stokes beam amplified by SBS and the excited modulated beam are compared in amplitude and phase as a complex transfer function by a vector network analyzer (VNA). The pulse response in time domain can be obtained by inverse Fourier transform of the complex transfer function. The frequency difference of the 


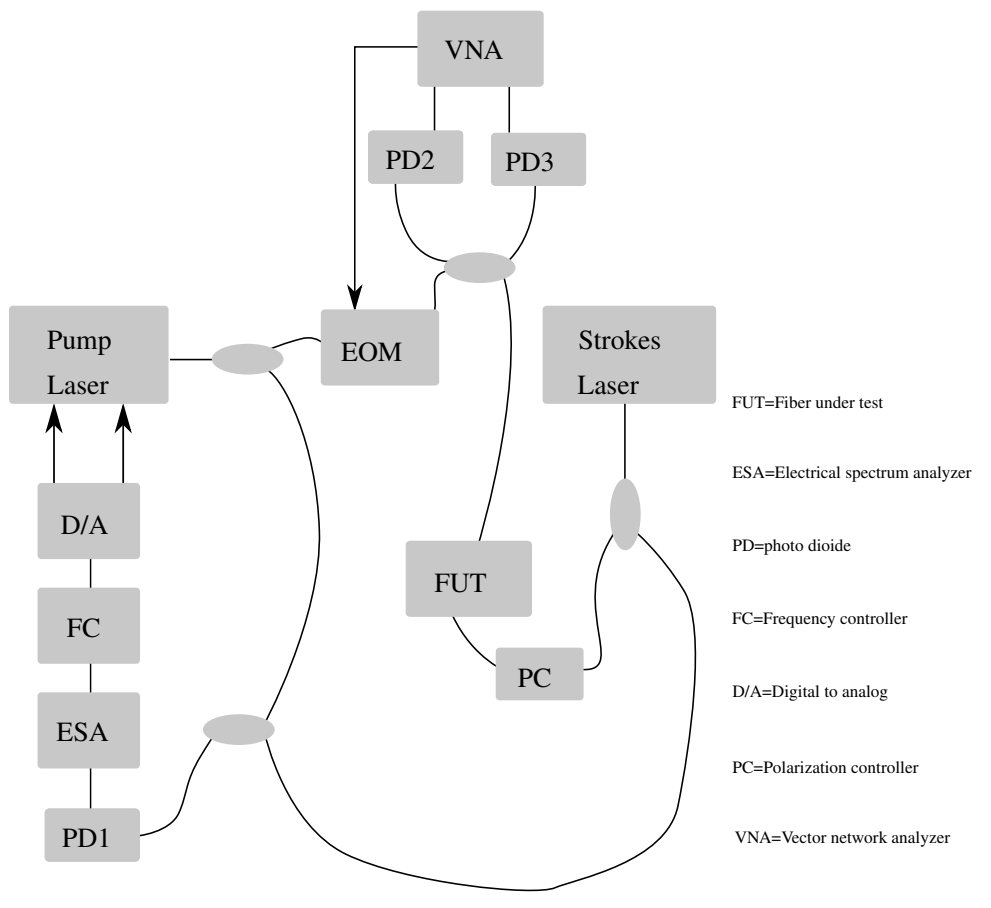

Fig. 6 Schematic diagram of Brillouin optical frequency domain analysis

two Nd:YAG lasers is tuned by a control loop. A photodioide (PD1) detects the beat of the lasers. An electrical spectrum analyzer measures the beat frequency. The results are sent to a frequency controller to calculate the offset from the desired frequency. An external digital-to-analog converter generates voltages to tune the resonator of the pump laser by means of the temperature element and piezoelectric actuator. Each spectral component of the pulse response is recorded separately and hence a narrow-banded filter can be used in the VNA. This filter enhances the dynamic range of the system but it takes much longer time for data acquisition because the bandwidth of the filter determines the settlement time of the system. The spatial resolution, measurement range, strain resolution and temperature resolution of a commercial BOFDA monitoring system are $1 \mathrm{~m}, 12 \mathrm{~km}, 2$ microstrains and $0.1^{\circ} \mathrm{C}$, respectively. The duration of data acquisition depends on the measurement distance. It ranges from 30 seconds for $200 \mathrm{~m}$ fiber to 5 minutes for $12 \mathrm{~km}$ fiber. 


\section{Civil Engineering Applications of the Optical Fiber Sensors}

\subsection{Highway Structures}

Bridges and highways are important infrastructures. Any closure may lead to heavy traffic congestion and enormous economic and social costs. Many optical fiber sensors have been installed on bridges and highways around the world. They monitor the global and local mechanical behaviors of bridges as well as environmental parameters such as temperature, pH level, humidity and chloride content. Point sensors are applied at critical components of the bridge such as bridge cables, anchorages, bridge decks, piers and pavements. Integral sensors are installed to measure the curvature and deflection of the bridge, as well as the onset of cracking. Distributed sensors are applied on the pavement to monitor the impact stress wave generated by high speed vehicles.

\subsubsection{Bridges}

When a bridge designed many years ago cannot satisfy the current traffic capacity, an economic solution is to retrofit the existing structure instead of building a new one. The North and South Versoix bridges in Switzerland were typical examples of bridge upgrade. They were two parallel twin bridges, each one supporting two lanes of the Swiss National Highway A9 between Geneva and Lausanne. The bridges were classical parallel prestressed concrete beams structures supporting a concrete deck and two overhangs. In order to support a third traffic lane and a new emergency lane, the exterior beams were widened and the overhangs extended. The construction progressed in two phases: the interior and the exterior overhang extension. The first phase began by demolishing the existing interior overhang followed by the reconstruction of a larger one. The second phase consisted of demolishing the old exterior overhang, widening the exterior web and rebuilding a larger overhang supported by metallic 


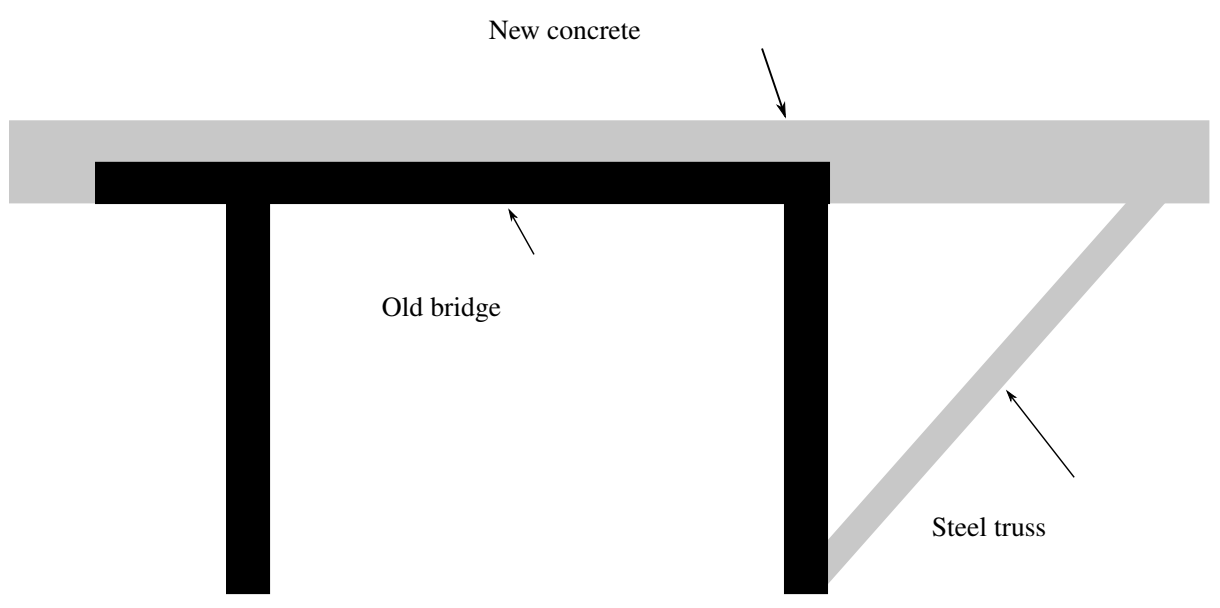

Fig. 7 The Versoix Bridge

beams (Fig. 7). More than a hundred long-gauge SOFO interferometers were installed on the Versoix bridges in order to monitor the deformation due to differential shrinkage between the old and new concrete. Also, the monitoring demonstrated how the deformation along the longitudinal direction of the bridge can be used to estimate the vertical deflection of the beam $[?, ?, ?]$. With a pair of SOFO interferometers installed on the compression side and tension side at the same location (Fig. 8), the local averaged curvature can be computed. The vertical deflection of the beam can be estimated by integration based on appropriate boundary conditions.

In 1997, the Waterbury bridge in Vermont, USA was rehabilitated and fiber Bragg grating (FBG) strain sensors, speckle pattern based vibration sensors [?] and chloride sensors [?,?] were embedded in the bridge deck [?]. There were three different approaches for the installation of FBG strain sensors. The first approach was to cast the bare FBG sensors in a relatively malleable plastic compound (Castolite) and metal pin was placed to locate the central position of the FBG sensor. The completed Castolite FBG sensor was mounted on the steel reinforcement before concrete casting. The second approach was to glue the FBG strain 


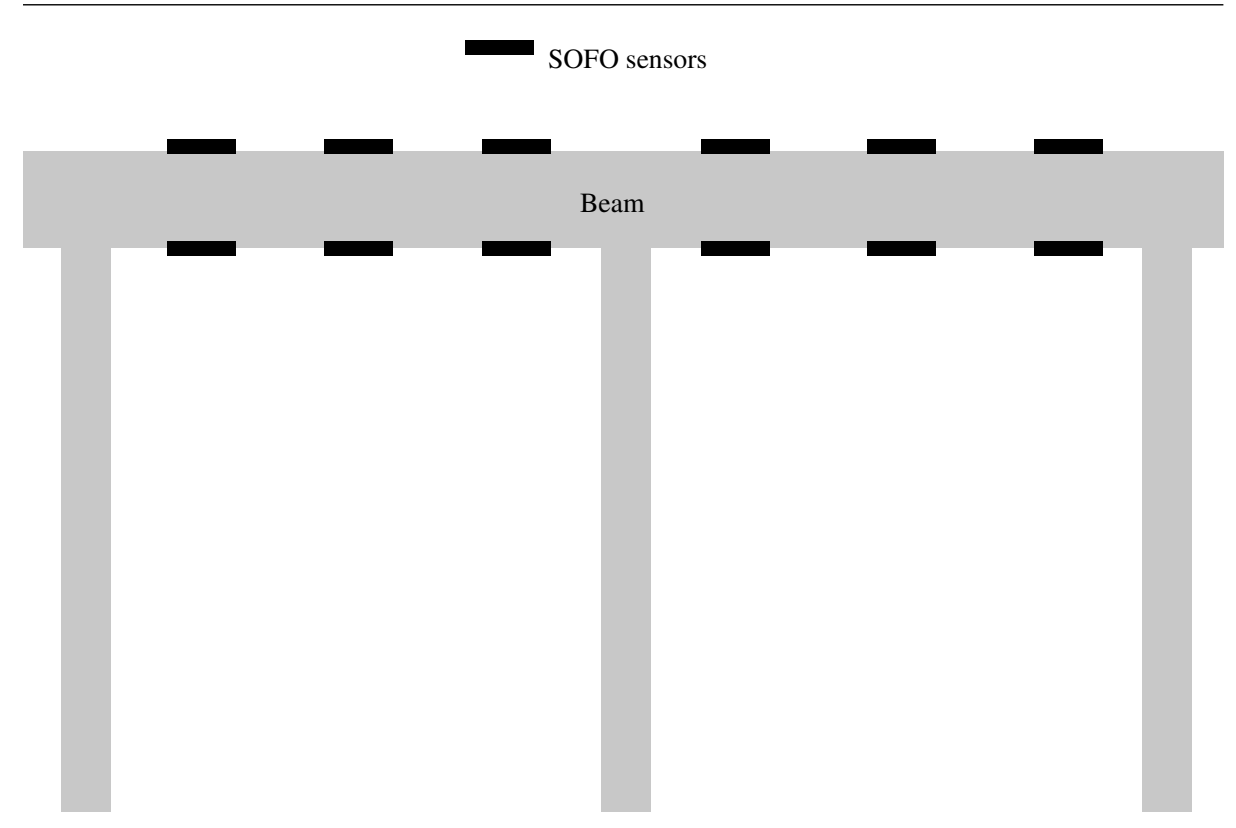

Fig. 8 The schematic diagram of the position SOFO interferometers on Versoix Bridge

sensor to a $1 \mathrm{~m}$ long epoxy-coated steel reinforcement by the same glue used for electrical strain gauges. The epoxy coating of the steel reinforcement is to protect the steel from corrosion. The instrumented $1 \mathrm{~m}$ long rebar was physically attached to the steel reinforcing system of the bridge deck. The third approach was to attach the FBG strain sensors directly on the bridge deck reinforcements by epoxy. The advantage of the second approach over the third approach is that the second approach can be performed in well controlled environment while the quality of the third approach depends on weather conditions. The sensing principle of the 36 installed chloride sensors relies on the color change (from milky white to pink) when chemical reaction occurs between the water transported chloride ion and the sol-gel film of the sensor. By varying the chemistry of the sol-gel, the range of the chloride threshold can vary from $20 \mathrm{mg} / \mathrm{L}$ to over $2300 \mathrm{mg} / \mathrm{L}$. The vibration sensor consists of a coherent laser light source, a multimode optical fiber and a photo detector. While the total intensity traveling through the optical fiber remains the same, the intensity of individual mode varies with the 
variation of phase change and it shows different speckle patterns at the cleaved fiber end due to interference among different modes (Fig. 9). By inserting an opaque annulus in front of the photo detector, the light intensity detected by the photo detector changes with the variation of the phase difference induced by the mechanical strain in the sensing region. When the intensity of the laser source varies sinusoidally, it is possible to shift the frequency of the vibrating signal into a less noisy region at low frequency range as in civil engineering infrastructures. In this project, all the lead cables and the access boxes were embedded in concrete. The access boxes were filled with paper to absorb moisture and keep the cables out of concrete. They were excavated after removing the wooden formwork. All sensors survived and showed reasonable values after concrete pouring and excavation of the access boxes.

FBG sensors have also been employed to monitor the internal strain of (i) the box girder of a prestressed concrete bridge across the Ring Canal in Ghent, Belgium and (ii) the trough girder of a railway bridge along the rail track Gent-Moeskroen, Belgium during post-tensioning, proof load tests and in service load. However, as bare FBG sensor is too fragile to be embedded directly into the structure, different kinds of packaging techniques were developed. One approach was to attach FBG sensors on a steel reinforcing bars to be embedded inside the structure [?]. The sensing bar consisted of a FBG strain sensor and a thermocouple for temperature compensation. A narrow groove was notched on the bar and the FBG sensor was attached into the groove with adhesive at the middle of the bar while the thermocouple was bonded on the opposite side (Fig. 10). The test results showed that the strain measured by the sensing bars agreed with the results from the mechanical deformeter (demec).

Another approach to package FBG strain and temperature sensors is to embed the sensors into fiber reinforced polymer (FRP) (Fig. 11). The strain sensors can measure both tensile and compressive strain with high signal-to-noise ratio. Strain up to 5000 microstrains with 


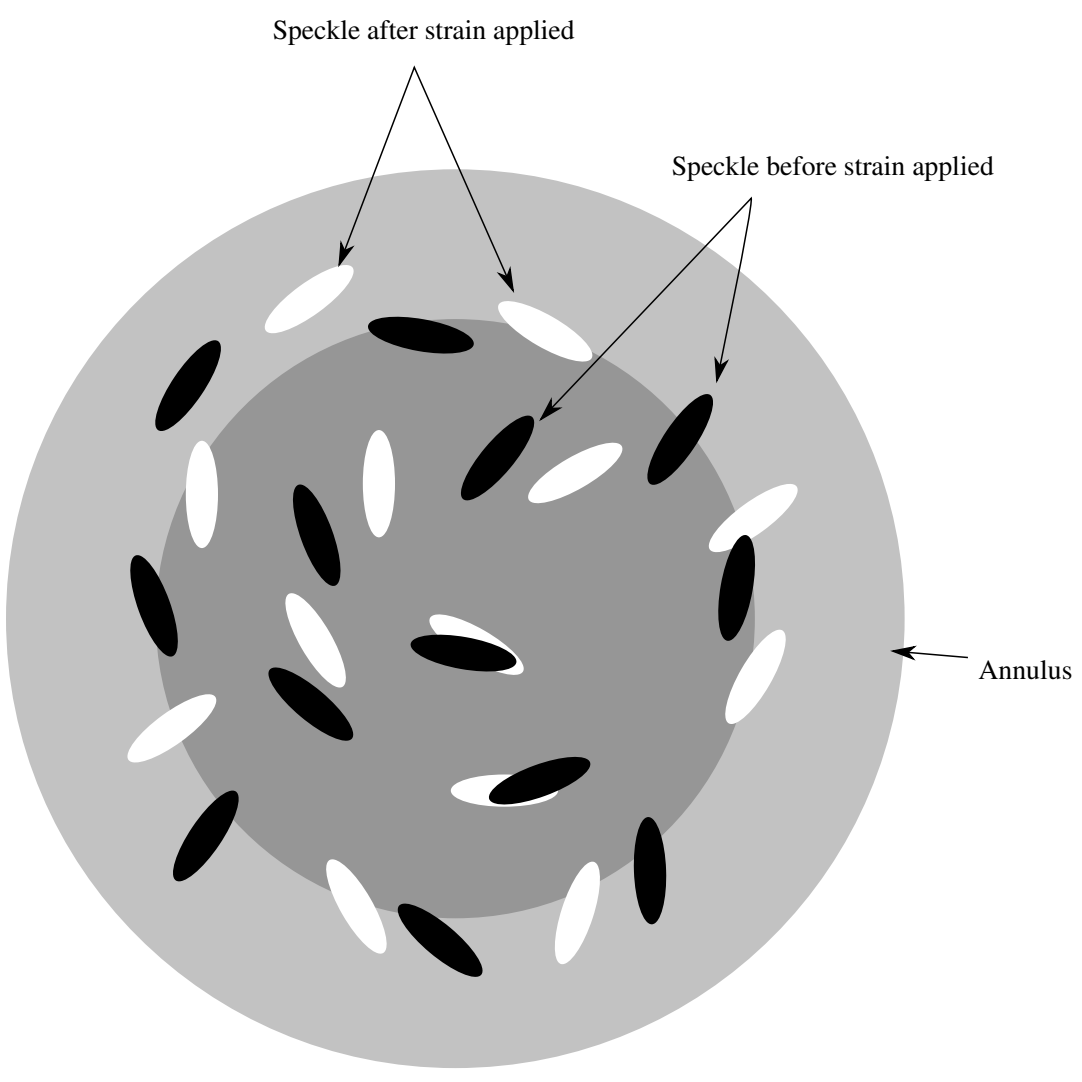

Fig. 9 Different speckle patterns at the cleaved end of a multimode optical fiber

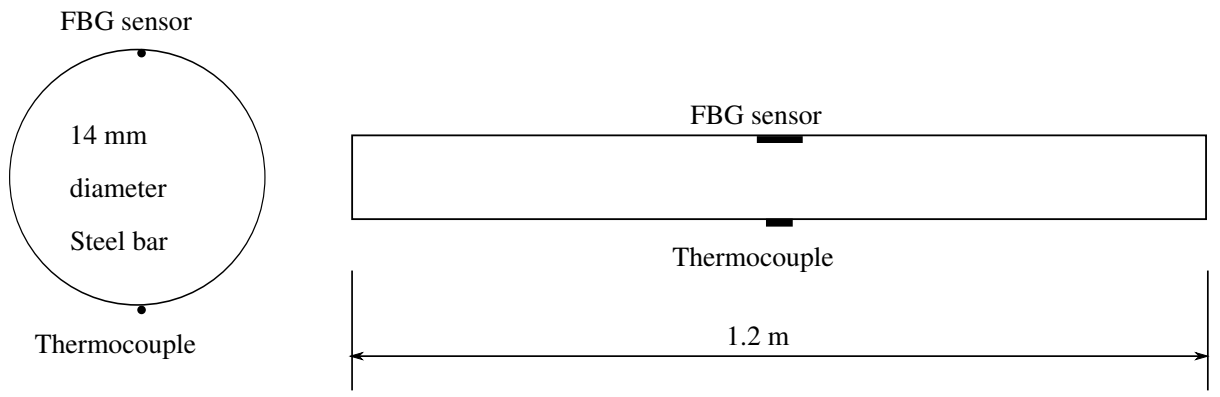

Fig. 10 A sensing steel reinforcing bar with a FBG strain sensor and a thermocouple

1-2 microstrains resolution can be measured [?]. This packaging method has been validated extensively in Shangdong Binzhou Yellow River Highway Bridge in Mainland China, which was open for traffic in July 2004. The bridge girder is made of prestressed concrete supported 
(a)
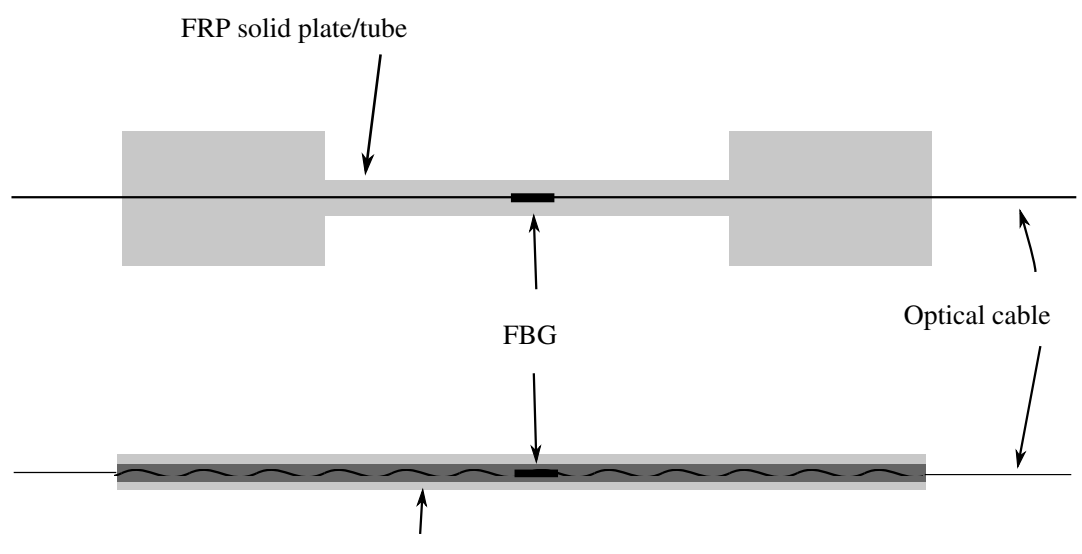

FRP hollow tube

Fig. 11 Schematic diagram of pointwise FRP packaged FBG sensors. (a) strain sensor, (b) temperature sensor

by 200 stay cables. The entire length of the bridge is $1698.4 \mathrm{~m}$ [?]. The maximum allowable truck load is 120 tons, which exceeds the maximum design load specified in the code of practice in Mainland China. The bridge was heavily instrumented with 1688 FBG strain sensors on the girders and the towers near the joint positions, as well as 180 FBG temperature sensors. The survival rate of those FBG sensors was about $75 \%$. The FBG strain sensors measured the longitudinal strain and transverse strain of the girder to estimate the effective prestress level and the strain near the cable anchorages.

Apart from monitoring the state of the bridge components during operation, the FBG sensors can also be used in monitoring the strain development of different components during construction stage. In October 2004, FBG strain and temperature sensors were installed in the foundation of the piers of the Third Nanjing Yangtze Bridge [?]. They monitored the internal temperature and internal strain of the mass concrete of the foundation during construction and operation.

When degradation occurs in cable stayed or suspension bridges, the load distribution among the cables will be changed. By continuously monitoring the tensile stresses at the 
cables induced by traffic load and temperature variation, any abnormal variation may indicate damage and fatigue of the bridge structure. The point sensor in Fig. 11 is not appropriate for installation on the cable. A smart cable was therefore developed to measure the load carried by the cable (Fig. 12). The optical fiber and the glass fibers were dragged by tension in parallel (Fig. 13) [?]. Resin was applied and then the composite solidified through heating in a furnace. The resulting FRP-FBGs consisted of an optical fiber at the center of the cross section and the FBG sensors were located at the pre-specified positions. The strain of the FBG sensors would be the same as the surrounding glass fibers. To find the actual mechanical strain, additional temperature sensors should be employed (either by using FBG temperature sensors in Fig. 11b or thermocouple). In the smart cable, the FRP-FBG cable replaces the central wire of a typical 7-wire steel cable (Fig. 12). Since the stiffness of FRP is much lower than steel, the load capacity of the smart cable is assumed to be six in seven of the original steel cable. The smart cables have been tested extensively in Sichuan Erbian Arch bridge [?,?] and Hunan Maocaojie Bridge at the arch suspenders and tie cables [?].

Due to excellent mechanical strength and corrosion resistance, carbon fiber reinforced polymer (CFRP) wires may replace steel cables in bridge construction. Two bridges in Switzerland with different types of CFRP cables were instrumented by FBG sensors [?]. For the Storchenbrücke cable stayed bridge in Winterthur, Switzerland, one of the twelve usual steel cable pairs were replaced by CFRP cables. Each cable is $35 \mathrm{~m}$ long and consists of $2415 \mathrm{~mm}$ diameter CFRP wires in a hexagonal structure and seven FBG sensors were installed. Three FBG sensors were attached at the circumference and spaced by $120^{\circ}$ relative to the center of the cable to measure the average strain as well as the strain gradient inside the cable. The strain level was between 1000 and 1500 microstrains. In addition, three FBG sensors were attached to three $30 \mathrm{~cm}$ long CFRP wire and another FBG sensor was bonded on an aluminum rod, which was bonded to the loaded wires in a way that it was insensitive to 


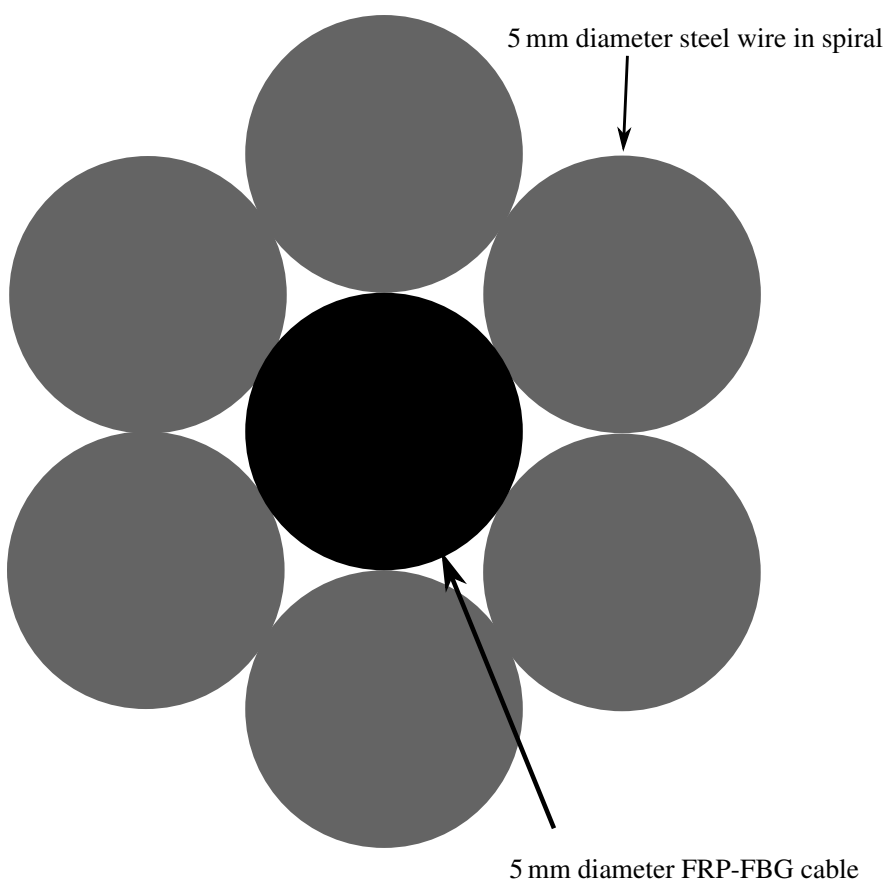

Fig. 12 Schematic diagram of the smart cable, which consists of a FRP-FBG cable at the middle and 6 steel wires in spiral

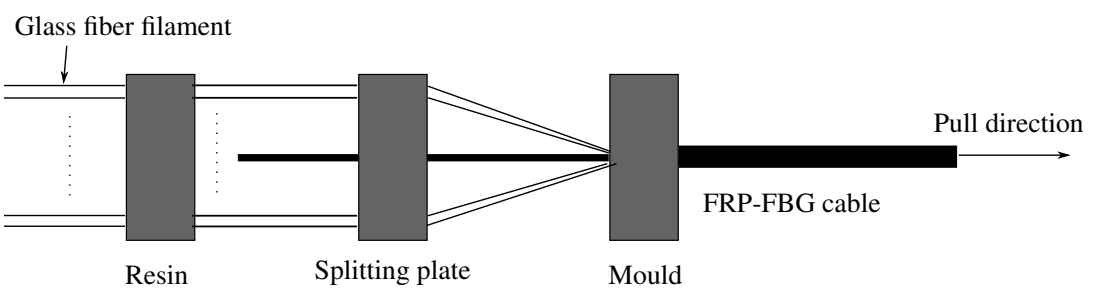

Optical fiber with FBG sensors at predefined positions

Fig. 13 Schematic diagram of the fabrication of FRP-FBG.

mechanical strain. The 4 mechanical strain insensitive FBG sensors served for temperature compensation and monitoring of long-term drift. By improving the data acquisition and wavelength evaluation techniques, the standard deviation of single strain measurement can be reduced from 7 microstrains to 2.5 microstrains. 
A new steel-concrete-composite footbridge near Luzern, Switzerland was constructed in 1998. Under the bridge deck, there are two $47 \mathrm{~m}$ long, $5 \mathrm{~mm}$ diameter CFRP prestressing cables consisting of 91 wires in hexagonal structure. About $1 \%$ to $1.5 \%$ prestress strain is expected. FBG sensors were embedded in the CFRP cables during fabrication to monitor the strains at the different cross sections of the 4 anchorage heads as well as the prestressed cables. Each CFRP wire consists of 30 rovings with each containing $120007 \mu$ m diameter carbon filaments. The optical fiber was placed at the center of the filaments. Then, the CFRP wires with the sensing fiber were pulled through a pultrusion machine and two ovens at temperatures between $170^{\circ} \mathrm{C}$ and $190^{\circ} \mathrm{C}$. To fabricate a mechanical strain insensitive $\mathrm{FBG}$ sensor for temperature compensation, the uncoated optical fiber was embedded in a Teflon tube with inner and outer diameters of $0.3 \mathrm{~mm}$ and $0.5 \mathrm{~mm}$, respectively. The reason of removing the fiber coating inside the Teflon tube is to improve the reproducibility, since the coating may have different thermal expansion coefficient with the silica fiber, degrades under high humidity and exhibits stick-slip effect. To connect the embedded optical fiber to an external measurement system, it was located by elevating the temperature up to $650^{\circ} \mathrm{C}$, at which the CFRP wire oxidizes while the optical fiber survive. The optical fiber was then spliced with a lead cable. The splice point was protected by a protective tube filled with epoxy and reinforced by Kevlar at the fiber exit point for better anchorage. All strained sensors showed reliable values and only one strained sensor debonded during the half year on-site measurement.

The major drawback of FBG sensor is the sensitivity to both mechanical and temperature induced strain. In conventional design, temperature sensors were placed alongside the mechanical strain sensor. A special packaging technique for FBG strain sensor with self temperature compensation by using two FBG sensors has also been developed [?]. Two FBG sensors were connected in series (about $30 \mathrm{~mm}$ separation) and sandwiched between two 


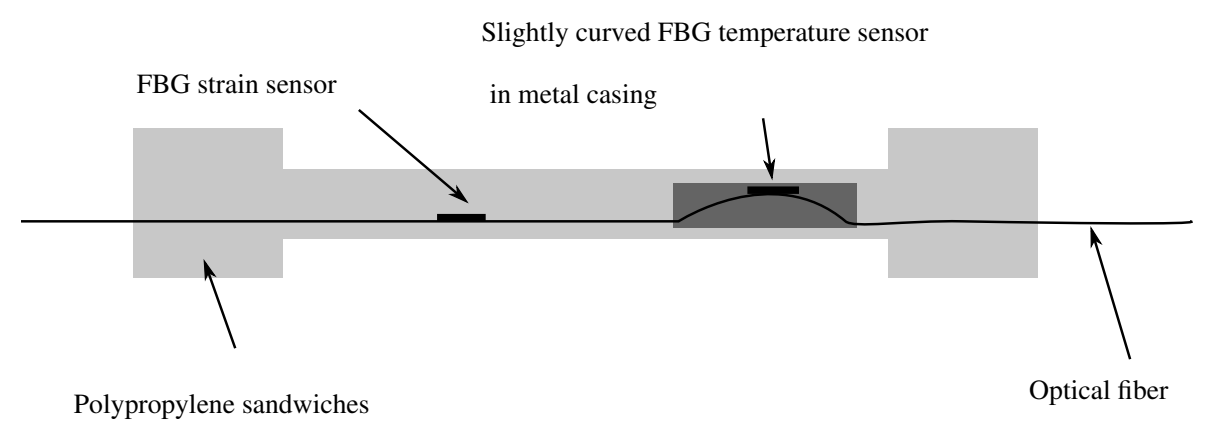

Fig. 14 Schematic diagram of a FBG strain sensor with self temperature compensation

plastic (polypropylene) slabs (Fig. 14). The mechanical strain sensitive FBG sensor was fixed by cyanoacrylate adhesive. The other sensor was isolated from mechanical strain by a special packaging scheme. The grating was slightly bent and enclosed in a thin metal tube in order to protect the grating and prevent the transfer of mechanical strain to the grating. The packaged sensor, which was $80 \mathrm{~mm}$ in length and $6 \mathrm{~mm}$ in width, were installed successfully in Monkstown Bridge in UK, which is a flexi-arch bridge, to monitor the strain development during construction and operation.

Loss of prestressing force of bridge structures has significant consequence on safety and serviceability. The immediate loss due to elastic deformation can be estimated precisely. However, it is difficult to predict the time dependent loss from several intertwined factors such as concrete creep, steel relaxation, concrete shrinkage and thermal effects. A smart steel strand similar to the 7-wire smart cable in Fig. 12 was developed with a single mode optical fiber to monitor the prestressing force during operation $[?, ?, ?]$. The central steel wire is replaced by a FRP cable with single mode silica optical fiber at the center of the rod (Fig. 13). The strain distribution along the smart steel strand was monitored by using Brillouin optical time domain analysis (BOTDA). Unlike Brillouin optical time domain reflectometry, the sensor has to form a closed loop for measurement. Since the strain is averaged within the 


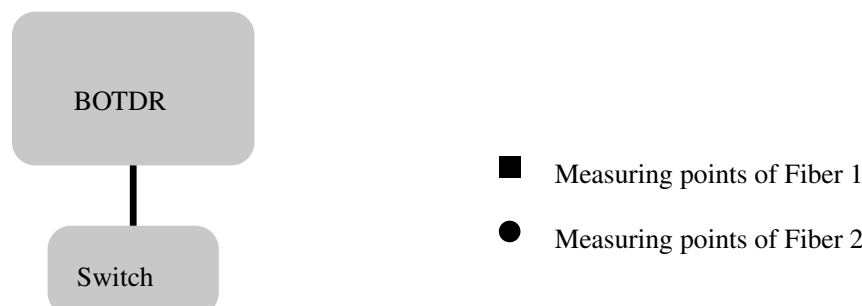

Fiber 1

Length $L_{1}$

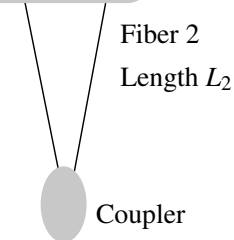

Fig. 15 Schematic diagram of a equidistance difference measuring method

spatial resolution and the duration of data acquisition is several minutes, it is only suitable for quasi-static measurement for long sensing distance. By the laboratory calibration tests, the coefficients of strain-to-Brillouin frequency shift (BFS) related to either mechanical or thermal effects depends on both the gauge length and the spatial resolution [?]. When the gauge length is shorter than the spatial resolution, the measured averaged strain includes the strain in the region beyond the gauge length.

The spatial resolution of commercial BOTDR/A model is about $1 \mathrm{~m}$. The high strain gradient near stress concentration region is averaged and may not be identified from the BFS in time domain. An innovative and simple method to identify any local damage is shown in Fig. 15. Two optical fibers with different lengths $L_{1}$ and $L_{2}$ are connected to an optical switch. When $\left|L_{1}-L_{2}\right|$ is less than the spatial resolution, it can improve the density of measurement points with fixed spatial resolution. If there is local stress concentration, a significant deviation is shown near the stress concentration region in the plot of the difference between the two strain measurements. 
Strain induced by temperature change can be compensated by two optical fibers embedded in the same matrix. With one of them sensitive to both mechanical and thermal strain $(B 1)$ while the other sensitive only to thermal strain (B2), the mechanical induced strain can be deduced from Equation 5 with a single calibration coefficient $\left(K_{\mathcal{\varepsilon}}\right)$ [?].

$$
\Delta \varepsilon=\frac{\Delta v_{B 1}-\Delta v_{B 2}}{K_{\varepsilon}}
$$

where $\Delta \varepsilon, \Delta v_{B 1}, \Delta v_{B 2}$ and $K_{\varepsilon}$ are the mechanical induced strain, BFS of sensor $B 1$, BFS of sensor $B 2$ and the coefficient of strain-to-BFS, respectively.

The sensing capability can be enhanced by incorporating FBG strain sensors to provide precise strain measurement at critical positions such as the anchorage points and perform dynamic strain measurement with sampling rate in range of kilo-hertz. In practical measurement, the FBG interrogator and BOTDA can be coupled as shown in Fig. 16. From the tests in [?], when the Bragg wavelength does not overlap with the wavelength of the laser of the distributed sensor, there is no noticeable interaction between FBG sensors and BOTDA. By incorporating FBG sensors with BOTDA measurement, the mechanical and temperature induced strain can be decoupled [?]. Assume there is no coupling effect between the mechanical and thermal induced strain of both sensors, the change in the Bragg wavelength $\left(\Delta \lambda_{B}\right)$ and the Brillouin frequency shift $\left(\Delta v_{B}\right)$ are related to the change in mechanical strain $(\Delta \varepsilon)$ and temperature $(\Delta T)$ through the sensitivity coefficients $C_{\varepsilon \lambda}, C_{T \lambda}, C_{\varepsilon v}$ and $C_{T \nu}$. The mechanical induced strain and change in temperature can be obtained uniquely by solving Equation 6.

$$
\left[\begin{array}{c}
\Delta \varepsilon \\
\Delta T
\end{array}\right]=\left[\begin{array}{ll}
C_{\varepsilon \lambda} & C_{T \lambda} \\
C_{\varepsilon v} & C_{T v}
\end{array}\right]^{-1}\left[\begin{array}{l}
\Delta \lambda_{B} \\
\Delta v_{B}
\end{array}\right]
$$




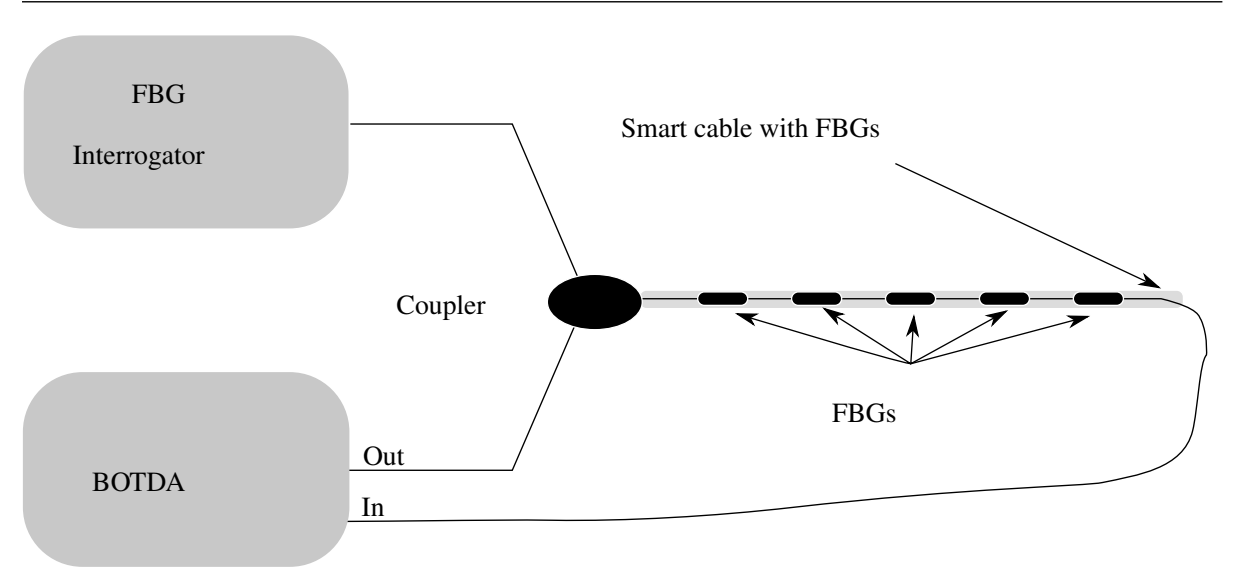

Fig. 16 Schematic diagram of a coupled FBG strain sensors and BOTDR

Götaälvbron, the bridge over Göta river, was built in the thirties and is now more than sixty years old. The bridge is more than 900 meters long and consists of a concrete slab supported on nine steel continuous girders, and nearly 40 columns. During maintenance, a number of cracks were found in the steel girders, notably in zones near columns where there are hogging bending moment. Two reasons can be proposed for the cause of steel cracking: fatigue and mediocre quality of the steel. The bridge authorities decided to keep the bridge in service for another fifteen years, but in order to increase the safety and reduce uncertainties related to the bridge performance, an integrity monitoring system is mandatory. The aim of the monitoring system is to detect and locate new cracks that may occur due to fatigue and to automatically send warning messages to responsible engineers. The main issue related to the selection of the monitoring system is that the total length of all nine girders is over $8 \mathrm{~km}$. It was therefore decided to monitor the five girders that are most heavily loaded (with total length of $4.5 \mathrm{~km}$ approximately) and logically applied a fiber optic distributed sensing system based on Brillouin scattering and tape-shaped sensors. It is the first time a truly distributed fiber optic sensing system was employed in such a large scale to monitor bridge integrity [?]. 


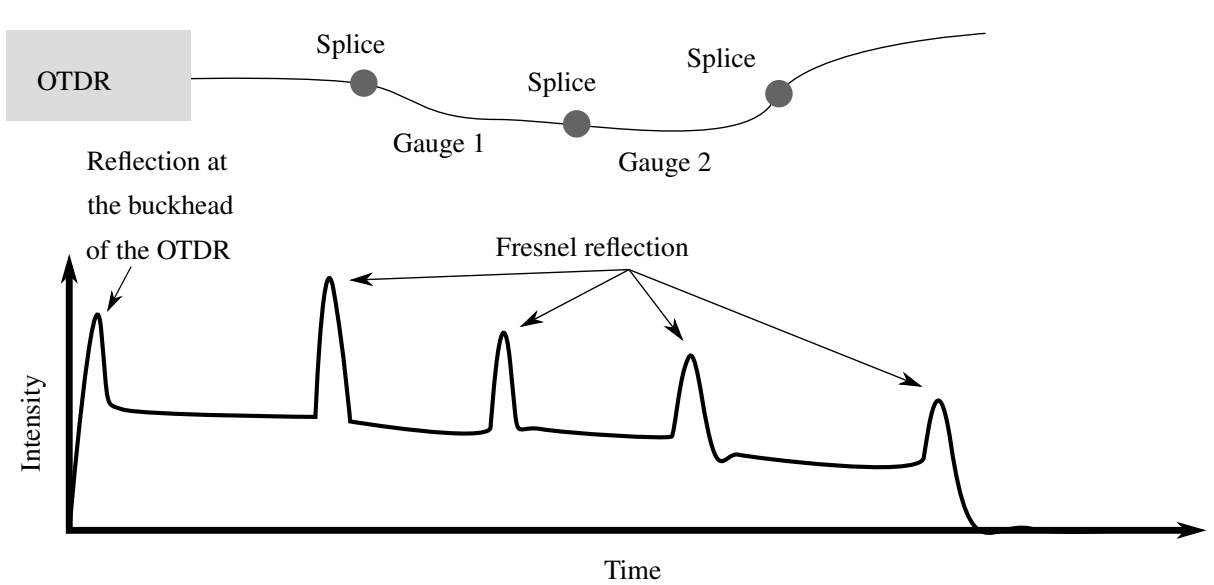

Fig. 17 Schematic diagram of an OTDR based multi-gauge crack sensor

By using optical time domain reflectometer (OTDR), several intensity based sensors can be multiplexed in time domain. A multi-gauge crack sensor based on the intensity of Fresnel reflection was developed [?,?]. An optical fiber was sliced into several segments by precision cleaver, each representing a gauge length (Fig. 17). The cleaved segments were spliced along one line to form the quasi-distributed sensor. By using OTDR, the intensity of the Fresnel reflection at each splicing point was measured. The sensor showed linear relation between the integral crack width and return loss of the corresponding segment. The sensor can be embedded into reinforced concrete structure and the loss was repeatable under fatigue loading.

Due to high inhomogeneity of concrete, it is difficult to have priori-knowledge of exact crack location. However, the direction of crack opening can be predicted. A distributed fiber optic crack sensor based on OTDR was developed $[?, ?, ?]$ to monitor multiple cracks by a single optical fiber. With known crack opening direction, the optical fiber is aligned at an angle to the crack. To facilitate the installation, the optical fiber with predefined orientation is first embedded into a $2 \mathrm{~mm}$ thick polyester plate filled with sand to make the plate more brittle (Fig. 18). The sensor plate is attached onto concrete surface by epoxy. To embed 
the plate into concrete, coarse aggregates, which provide interlocking with concrete, are inserted before hardening of polyester plate. When crack in concrete opens, the sensor plate is cracked and the optical fiber has to bend in order to maintain continuity. Bend loss (and hence a drop in the intensity of the Rayleigh backscattered signal) is hence induced near the crack location (the lower graph of Fig. 18). The OTDR is used to measure the intensity of Rayleigh backscattered signal with respect to time. The slight decrease in backscattered signal is due to attenuation, while sudden signal loss corresponds to the opening of a crack. The amplitude of the signal loss is related to the crack opening size, optical fiber type and the angle between the optical fiber and the crack opening direction [?]. The location of the crack can be estimated from the arrival time of backscattered signal, as the speed of light in optical fiber is known. With this technique, several cracks can be monitored by a single optical fiber. The limitation of the sensor is the spatial resolution, accuracy and dynamic range of the OTDR. If the distance between two adjacent cracks are smaller than the spatial resolution of the OTDR, the losses from individual crack cannot be distinguished from the OTDR reading. The accuracy of the OTDR determines the uncertainty of crack size estimation. The dynamic range determines the maximum number of cracks that can be monitored because the signal intensity drops after traveling through each crack. The sensor prototype has been verified by experiments and field trials. The sensor was used in the loading test of Ovid Bridge in Sweden before its demolition, and the average crack opening obtained from the sensor was in reasonable agreement with direct measurements. For a field trial in Mongkok, Hong Kong, the crack sensor plate was successfully installed on a surface of a highway bridge pier. To improve the spatial resolution of low-end OTDR (about $3 \mathrm{~m}$ ), fiber loop (more than $10 \mathrm{~m}$ ) was inserted between two adjacent optical fiber segments. The sensors showed stable reading after two years in outdoor environment. In the laboratory, the sensor was further extended to monitor in-plane mixed mode crack opening [?]. 


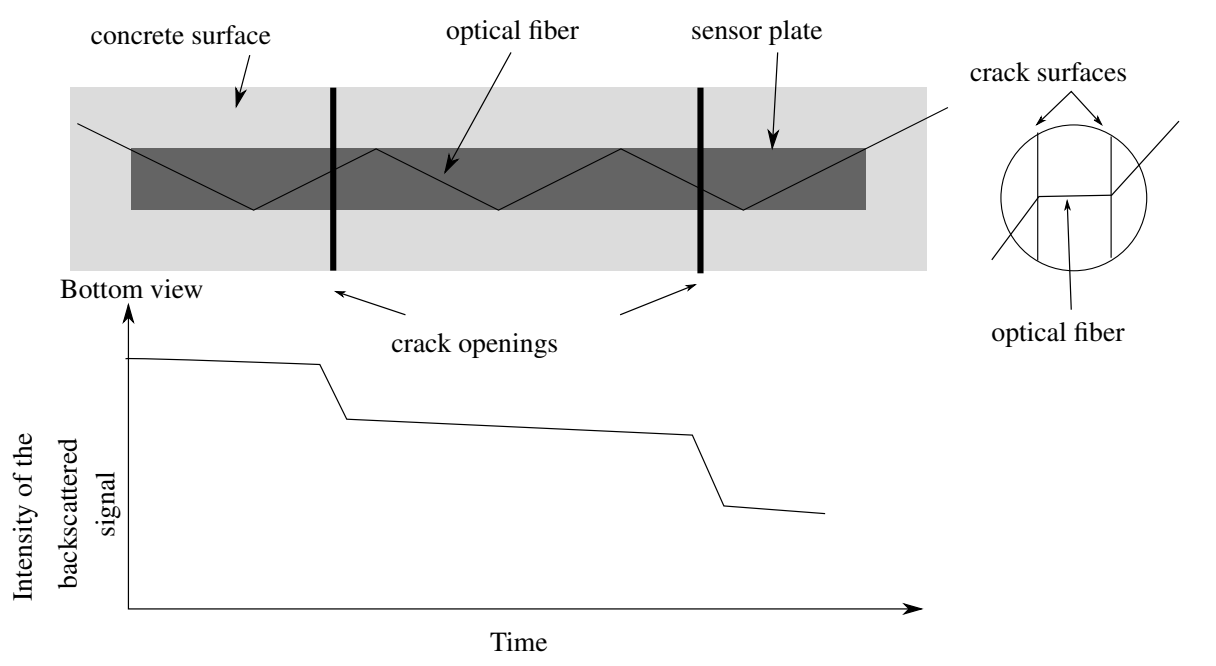

Fig. 18 Sensing principle of the OTDR based distributed crack sensor

The spatial resolution of Rayleigh scattering can be enhanced in a cost effective manner by using optical frequency domain reflectometry (OFDR) instead of optical time domain reflectometry (OTDR). Optical fibers were bonded on the lateral surface of a reinforced concrete beam as shown in Fig. 19. The spatial resolution is $6 \mathrm{~mm}$. From Fig. 19, the local stress concentration at tension side as well as compressive strain can be measured.

A pH sensor for cable corrosion was developed based on the absorption spectrum of evanescent field for tracking the drop of $\mathrm{pH}$ level [?]. The $15 \mu \mathrm{m}$ cladding is removed from a $600 \mu \mathrm{m}$ core diameter multimode optical fiber. The indicators used for the sensors are $\mathrm{pH}$ sensitive, non-fluorescent chromoionophores, such as methyl red, thymol blue, and thymolphtalein that are embedded into a polymethylmethacrylate (PMMA) matrix to reform the cladding of the optical fiber (Fig. 20). The PMMA cladding possesses several characteristics: (i) good bonding with the silica fiber core, (ii) permeable to water-born analysts, (iii) good bond with the dye molecules and (iv) lower refractive index than the fiber core to guide the light wave by total internal reflection. When there is a $\mathrm{pH}$ sensitive chromoionophore in the cladding, certain wavelength of the spectrum within the evanescent field is absorbed. The 


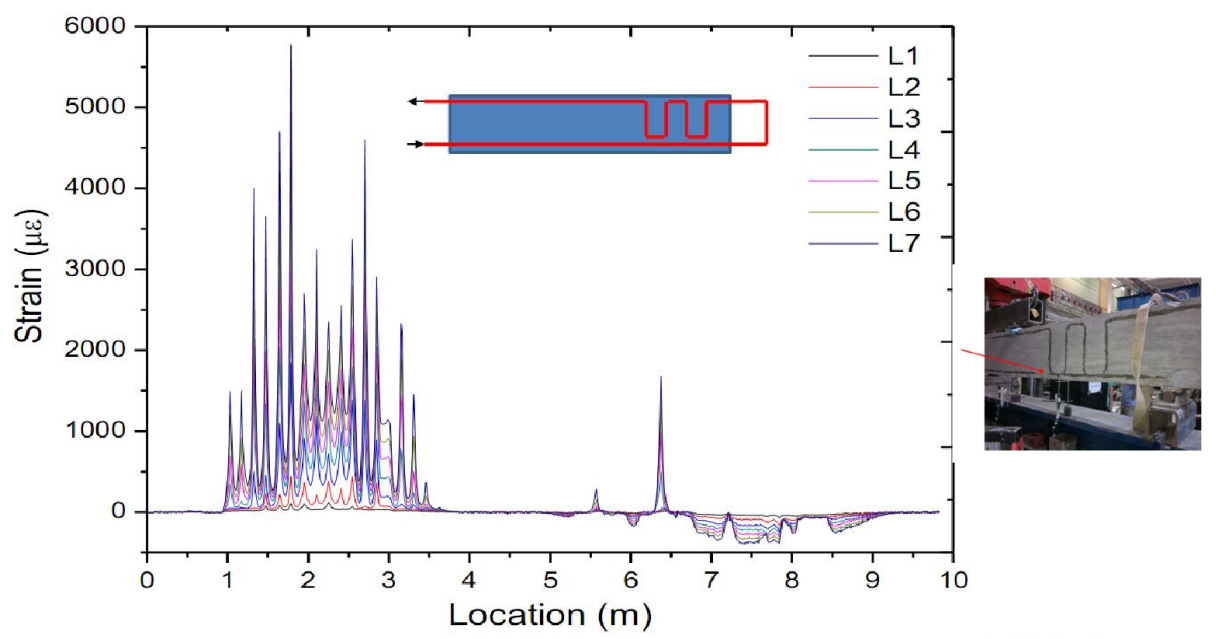

Fig. 19 Experimental results of distributed strain with $6 \mathrm{~mm}$ spatial resolution by optical frequency domain reflectometry

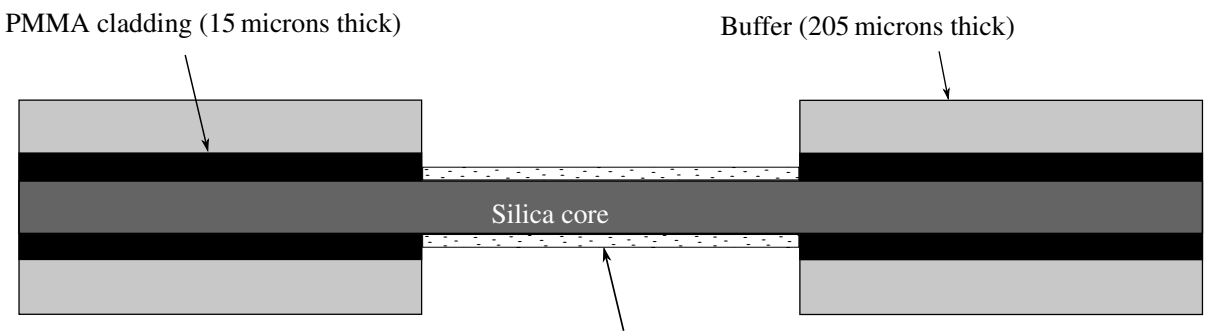

Recoated PMMA cladding

with chromoionphore

(10 microns thick, $65 \mathrm{~mm}$ length)

Fig. 20 Schematic diagram of a pH sensor based on the absorption spectrum of evanescent field

absorption spectrum depends on the $\mathrm{pH}$ value. The response time of this $\mathrm{pH}$ sensor is about 10 hours and it is determined by the permeability coefficient of cladding. By changing the material used for the cladding from PMMA with indicators to cellulose [?], which is a highly porous polymer, the ingress of the liquid phase moisture content for the bridge cables can be monitored. 
(a)

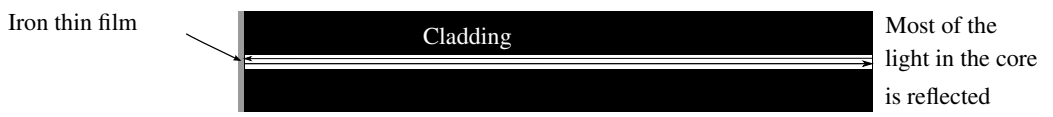

(b)

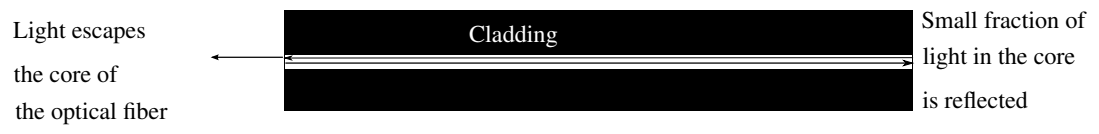

Fig. 21 Sensing Principle of the corrosion sensor based on Fresnel reflection. (a) Original, (b) at corrosive environment

A corrosion sensor for reinforced concrete structures was developed based on the variation of Fresnel reflection in corrosive environment [?]. The sensor is made by sputtering a very thin pure iron coating (about $200 \mathrm{~nm}$ ) on the cleaved end of a single mode telecommunication optical fiber (Fig. 21). The iron coating reflects the light as a mirror. When the surrounding environment becomes corrosive (either by chloride ion or carbonation), the coating is depleted and the reflectivity is significantly reduced. The sensor reflectivity can be measured by OTDR. To monitor the reflectivity of multiple sensors, an optical splitter can be placed between the OTDR and the sensors [?]. The sensor can be installed in existing or new structures. In a field trial test in Deep Bay, Hong Kong, 10 sensors were installed in two footbridge piers under aggressive marine environment. Two of them were controls installed at the non-corrosive locations, and they were still working over three years after installation. The projected durability of the sensors by accelerated life test and freezing-thawing cyclic test is about 70 years and 350 cycles, respectively. 


\subsubsection{Pavements}

Highway pavement consists of several layers from compacted soil to concrete/bitumen surface. The strain distribution along vertical layers and circumferential directions may change dramatically. The three-dimensional stress state governs the yielding and failure of the material at a particular point. As conventional FBG sensor can only measure the longitudinal strain, a three-dimensional embeddable strain sensor was developed [?]. Three FBG strain sensors were embedded in FRP with appropriate steel annular anchorage in three orthogonal directions. Fig. 22 shows the projection of the three-dimensional strain sensor on $\mathrm{x}-\mathrm{z}$ plane. The configuration along $\mathrm{y}$ direction is the same as that in the $\mathrm{x}$ direction. The gauge length in the vertical direction (z) was only $14 \mathrm{~mm}$ to prevent instability under the vertical compressive stress and limit the total height of the sensor to be less than onethird of the typical thickness of a pavement layer. However, the short gauge length might lead to inefficient strain transfer, and correction factor should be applied to the measured vertical strain. The size and shape of the anchorage were designed to minimize the stress concentration and maximize the strain transfer efficiency. The FBG three-dimensional strain sensors have been embedded into different layers of highway pavement of Tailai highway in Shandong Province, Mainland China to monitor the induced strain by a static heavy vehicle on pavement surface.

FRP reinforced concrete has been used in bridges and pavements to eliminate the corrosion problem of steel but the compressive strength of FRP can be significantly reduced by impact damage, which cannot be identified by visual inspection. Strain monitoring is a way to assess damage. In a field trial at Highway 40 East, Montreal, Quebec, Canada, Brillouin optical time domain analysis was used to measure the impact wave on concrete pavement induced by high speed vehicles $(>100 \mathrm{~km} / \mathrm{h})$ [?]. Standard telecommunication silica single 


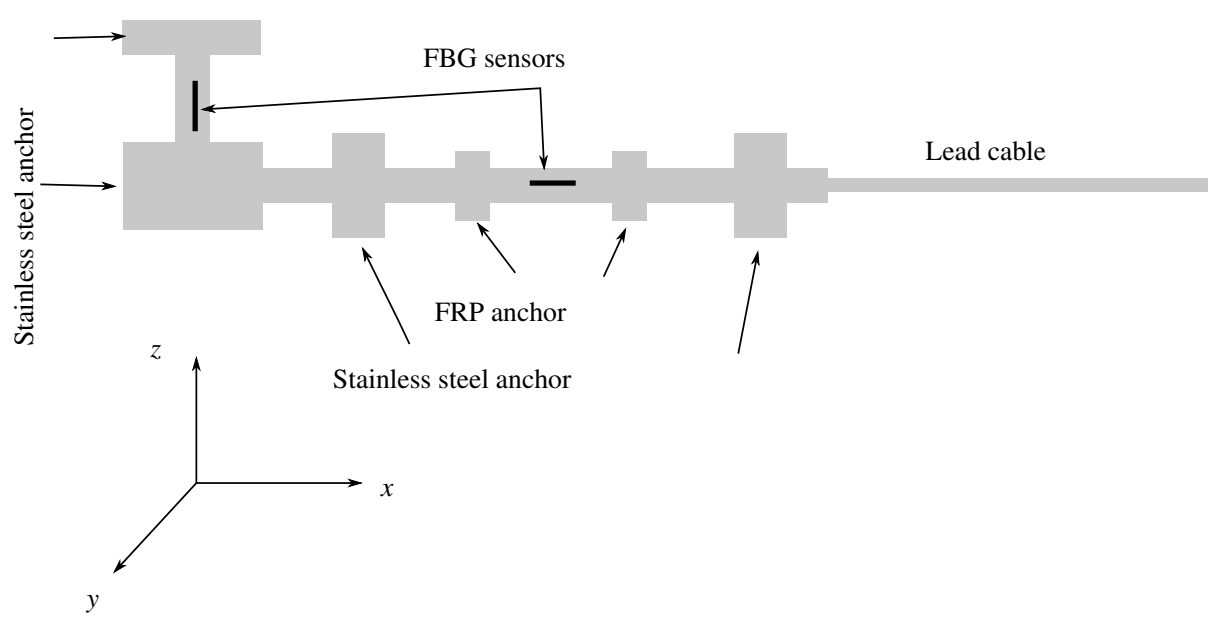

Fig. 22 Schematic diagram of FBG based 3D strain sensor

mode optical fiber was attached on the FRP bars, which were embedded in continuous reinforced concrete pavement slabs of the highway. The sensor can monitor both the static and dynamic impact strain. The distributed impact wave is monitored based on the change in polarization state induced by the local change in birefringence from the pressure wave. The response time is in the range of milliseconds and the maximum detectable frequency component is about $10 \mathrm{kHz}$ when the number of waveform averaging is kept at minimum. With this technique, there is a trade-off among the accuracy in strain measurement, spatial resolution and maximum frequency response. The field trial showed that the system could measure static strain to an accuracy of 50 microstrains with spatial resolution of $2 \mathrm{~m}$.

\subsection{Building Structures}

Building structures are mostly related to human activities. Ultimate or serviceability failure will lead to safety problems and inconvenience. When new innovations in materials, structural forms and construction technologies are employed in modern buildings, engineers may not 
be able to understand and anticipate the exact behavior of the structures during construction and operation. It is then desirable to have a continuous monitoring system to evaluate the symptoms of operational incidents, anomalies, deterioration that may affect operation, serviceability, safety and reliability of the structure. For historical buildings, the technical details of the structures are usually not available and the degree of deterioration is often not known. Structural monitoring can then be employed to facilitate the planning of appropriate management and maintenance scheme.

\subsubsection{Modern Buildings}

Singapore is a cosmopolitan city-state with many tall buildings. The Housing and Development Board (HDB), which is the public housing authority of Singapore, has been providing public housing for Singaporeans through a comprehensive building program. As part of the quality assurance of new HDB tall buildings, long-term structural monitoring of a new building project at Punggol East Contract 26 was started in 2001. This monitoring project was considered as a pilot project with two objectives: (i) to develop a monitoring strategy for column-supported building structures, and (ii) to collect data and provide information related to the behaviors and health conditions of this particular building type from construction to in-service operation. The project consisted of six nineteen-storey building blocks founded on piles and each block consisted of 6 units supported by more than 50 columns at ground level.

The specific aims of monitoring are to (i) increase the knowledge of the real structural behaviors, (ii) have better control of construction process, (iii) increase the safety during the service life, (iv) enhance the efficiency of maintenance activities and (v) evaluate the structural conditions after extreme events such as earthquake, typhoon or terrorist attack. The monitoring is performed at (i) local, column level and (ii) global, structural level. The ground columns, which are the most critical elements in the building, were selected for monitoring. 
Ten SOFO long gauge sensors with $2 \mathrm{~m}$ gauge length were embedded in each block to measure the vertical deformation of the columns and the readings were taken manually during construction [?].

After the pilot project, about 600 other similar buildings were instrumented in the same way in Sinpagopre with approximated 10 sensors for each building block. They probably constituted the world's largest deployment of fiber optic sensors to a fleet of similar structures.

It was the first time in Singapore to deploy optical fiber sensors on such a large scale structural monitoring on residential high-rise buildings and the results are beneficial to building designers by providing better understanding to the behaviors of building structures from construction to in-service operation. From the analysis of the collected data, it showed unexpected behaviors of columns during construction and these facilitate future research on the modeling of complex structures. In addition, with appropriate modeling and analysis software, it is possible to separate the measured total strain to time-independent component (elastic strain) and time dependent components (creep and shrinkage strain).

To understand the complex behaviors of modern buildings and structures, computational methods such as finite element modeling is often employed in the design stage. However, even sophisticated computer model involves many assumptions and uncertainties. As an example, Guangzhou New TV Tower (GNTVT) is a tube-in-tube structure with height of $610 \mathrm{~m}$. The structure consists of a reinforced concrete inner tube and a structural steel outer tube with concrete filled tube columns. The hyperbolic shape makes the structure aesthetically attractive but extremely complex in structural behaviors, especially the wind-structure interaction. Even the wind characteristic at the top level of the building cannot be reliably obtained. 120 FBG strain and temperature sensors were installed in GNTVT at 5 different levels for in-service monitoring as shown in Fig. 23 [?]. Specially packaged FBG strain sensors were welded on the surface of the structural steel of the outer tube columns in factory to measure both 


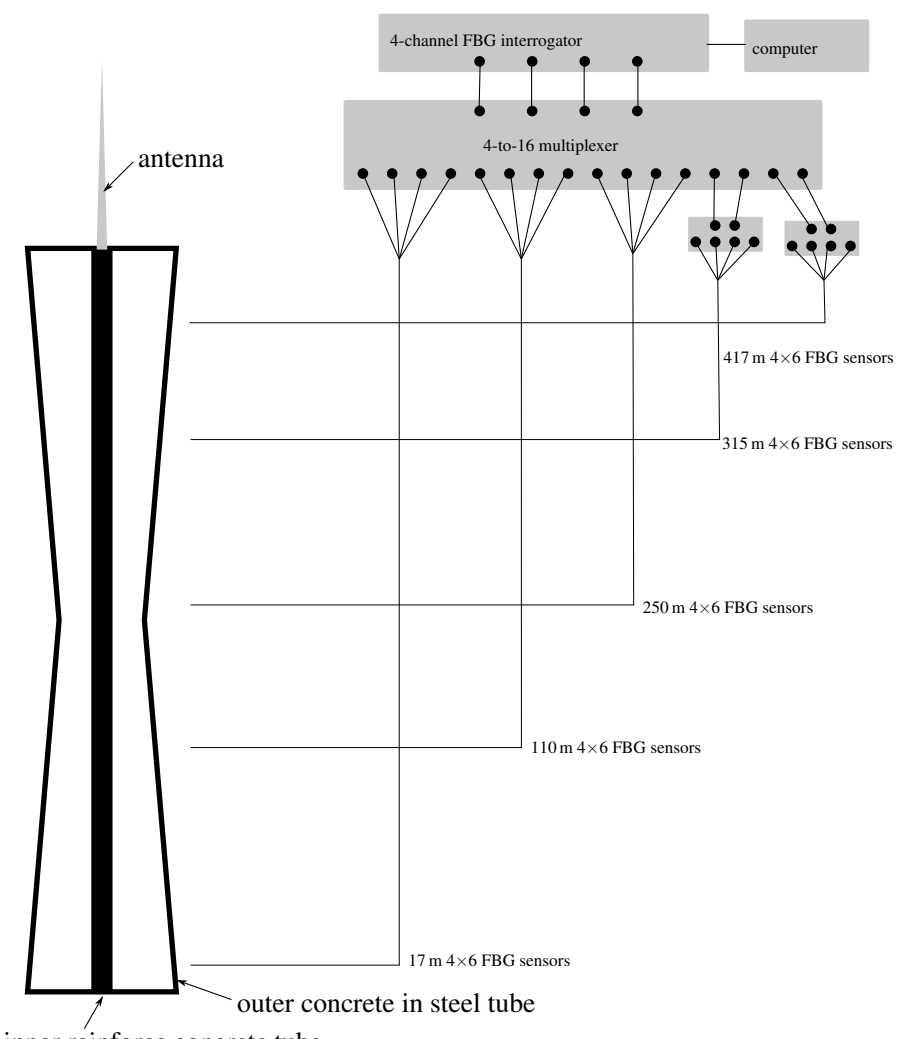

inner reinforce concrete tube

Fig. 23 Schematic diagram of the sensor allocations in Guangzhou New TV Tower

cumulative and dynamic strain. The sensors were packaged in a specially designed steel cage to prevent damage during welding. At each level, 24 FBG sensors were attached in 4 separated sensor chains. In each sensor chain, there were 4 FBG strain sensors and 2 FBG temperature sensors for temperature compensation. The strain and temperature resolutions are 1 microstrain and $0.1^{\circ} \mathrm{C}$, respectively. The maximum allowable strain is 2500 microstrains and the allowable working temperature range of the FBG temperature sensors is between $-40^{\circ} \mathrm{C}$ and $120^{\circ} \mathrm{C}$. Since the FBG interrogator can only handle 4 channels at $200 \mathrm{~Hz}$ sampling rate, all FBG sensor chains were connected to a 4-to-16 multiplexer. The major drawback of this approach is the reduction of sampling frequency on each sensor chain from $200 \mathrm{~Hz}$ to $50 \mathrm{~Hz}$. 
Other examples of FBG based structural health monitoring systems for modern buildings can be found in Shenzhen Civic Center, Vanke Center and Nation Aquatic Center in China [?]. The Shenzhen Civic Center consists of a space truss structure supporting a giant roof that is $486 \mathrm{~m}$ long, $154 \mathrm{~m}$ wide and $9 \mathrm{~m}$ in maximum thickness. $12 \mathrm{FBG}$ strain sensors were installed in the brackets and other key members to monitor the stress of the space truss and roof during service and under extreme loading condition such as typhoon. In Vanke Center, the floor area is more than $130000 \mathrm{~m}^{2}$ supported by 8 giant tubes and thick walls to provide maximum open space for a garden underneath. The main structure consists of a mixed frame-cable system. The steel cables transfer the vertical load from the steel truss girders to the concrete-in-steel columns. In order to monitor the stress of the key components during the construction and operation phases, 70 FBG strain sensors were installed on the steel truss girders, steel beams, concrete-in-steel columns and the giant columns. 16 FRP-FBG smart cables as shown in Fig. 12 were installed to monitor the tensile stress carried by the steel cables. In addition, 35 FBG temperature sensors were installed for temperature compensation of the strain sensors. The National Aquatic Center is a complex structural steel-membrane structures. Due to high redundancy of the steel structure, thermal induced stress may be significant. 240 FBG strain sensors were installed on the membrane roof and steel structures to monitor the fluid-solid interaction of the membrane, thermal induced stress on the steel members, as well as vibration under wind and other human activities. 40 FBG temperature sensors were installed for temperature compensation.

Comprehensive structural health monitoring was performed in the Berlin Central Railway Station, Germany [?]. The station is a complex structure made of concrete, steel and glass. At the station, the railway platform is supported by curved girders that need to be partially prestressed. Long guage SOFO interferometers were installed to measure the strain of the structure. The readout unit contains only single channel. For multichannel operation, the 
sensors are connected to a multiplexer and all data are transmitted to a central computer through Internet. The monitoring system is expected to work reliably over a long period of time and withstand all operational and environmental influences.

The distributed internal strain can provide valuable information for structural health evaluation. A typical building consists of beams, columns, slabs and walls. It is extremely difficult and costly to provide outlet for data logging from embedded sensors in each structural component. In addition, bare optical fiber is too fragile to survive concrete pouring and compaction. An innovative sensor installation method, called long-distance embedded optical fiber sensor (LEOFS), for measuring distributed internal strain sensing from the Brillouin scattering, was developed [?]. Aluminum/plastic tubes were fixed in the formwork before concrete casting to form a connected network. Single mode optical fiber with enlarged polymeric jacket ( $3 \mathrm{~mm}$ outer diameter) was laid through the tubes by air flow technique with air velocity between $20 \mathrm{~m}$ per minute and $50 \mathrm{~m}$ per minute. The one-time maximum length laid by air flow technique is about $500 \mathrm{~m}$. Then, the tubes were sealed by vacuum grouting technique using cementitious grout with properties of high fluidity, reasonable expansion rate and long setting time, made possible by using appropriate water content, water reducing agent, expansive agent, air entraining agent and retarding agent.

\subsubsection{Historic Buildings}

Historic structures may have an important role in the cultural heritage of society because of the age, architectural beauty, historical events, construction challenges at their era and many other reasons. However, with the old construction techniques and long time exposure to environmental conditions, there may be unknown degrees of deterioration that affects the structural functions. Also, records of the design and construction details are usually missing. For conservation of historic structures, it is desirable to have periodic/continuous monitoring 


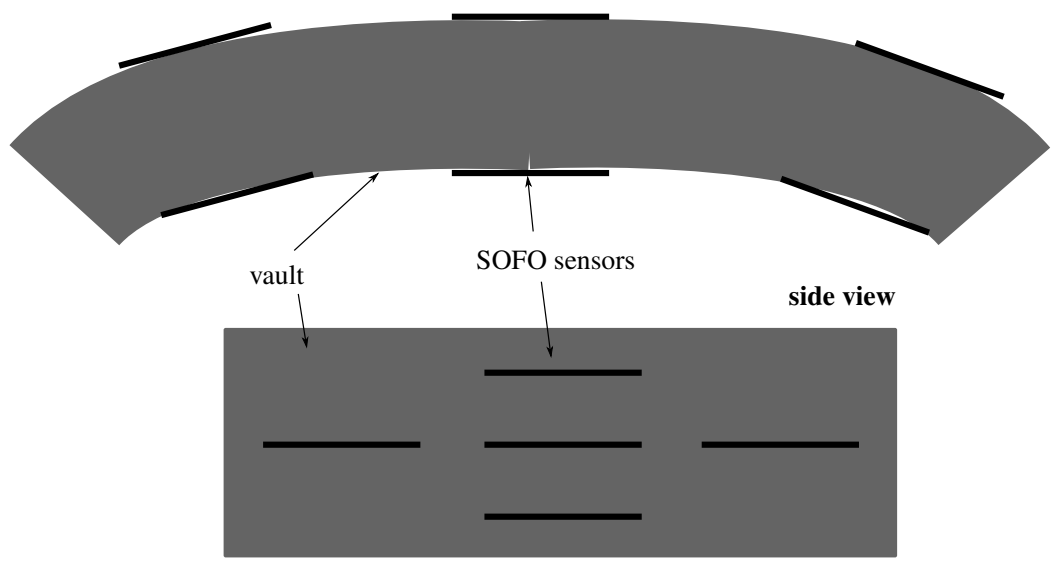

top view

Fig. 24 Long-gauge SOFO interferometers on the vault of San Vigilio church

systems to provide valuable information and accurate knowledge of the structural behavior for the development of proper management and maintenance scheme.

San Vigilio church is situated at Lugano lake in Switzerland. It was first constructed in the 16th and 17th century, while the frontal façade and portal was built in the 19th century. There were many significant cracks running along the center of the cylindrical vault of the church while some smaller cracks existed on the upper convex side of the vault. To assess the stability of the cracks, 10 long-gauge SOFO interferometers $(50-60 \mathrm{~cm}$ long) were mounted on both the top and bottom surfaces of the vault to monitor the cracks and the curvature (Fig. 24) [?]. The installed sensors are hardly noticed by uninformed observers.

Royal Villa in Monza, Italy, was built from 1777 to 1779. It was abandoned since 1900 and severe cracking was found along the barrel vaults of the central corridor at various levels. There was also significant degradation of the $18 \mathrm{~m}$ long truss wooden structures. Repair and conservation work was carried out to renovate the villa and transform it to a museum. Longgauge SOFO interferometers were installed between the walls orthogonal to the corridor axes 


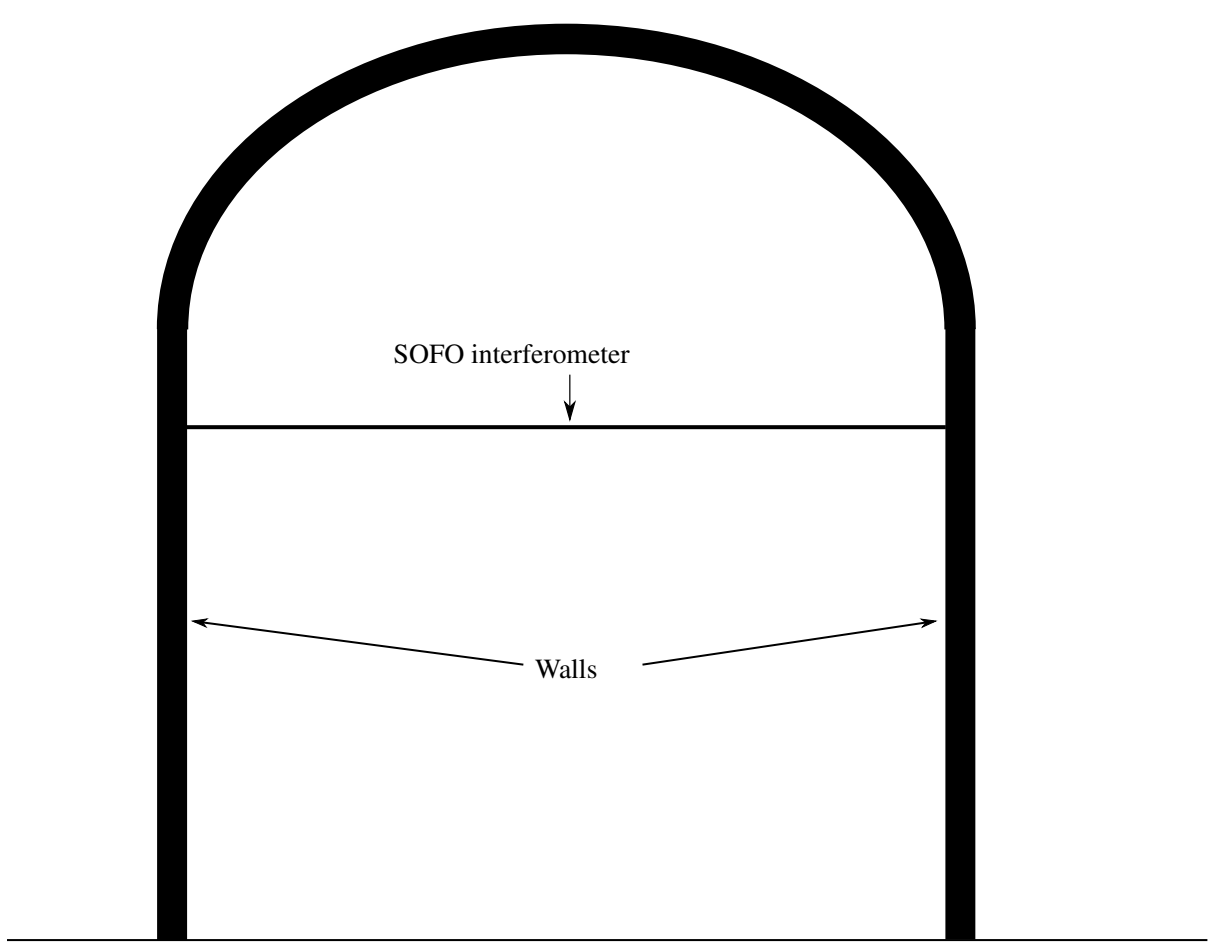

Fig. 25 SOFO interferometers in Royal Villa

to measure the global structural displacements (Fig. 25), while short-gauge length SOFO interferometers were installed to measure the propagation of the existing main cracks [?].

Bolshoi Moskvoretskiy Bridge in Moscow, Russia, was built in 1936 to 1937. As one of the main traffic routes to the Red Square, it is made up of three $100 \mathrm{~m}$ long reinforced concrete arches. The settlement of the center of the arches caused cracking in the stone walls near the abutments on both sides of the bridge. Also, chloride induced corrosion has led to cracking of the reinforced concrete arches. 16 long-gauge SOFO interferometers were installed on both upper and lower sides along the bridge to monitor the curvature continuously in both horizontal and vertical directions as well as deformation shape [?]. The data was sent to a central control station through telephone line. 


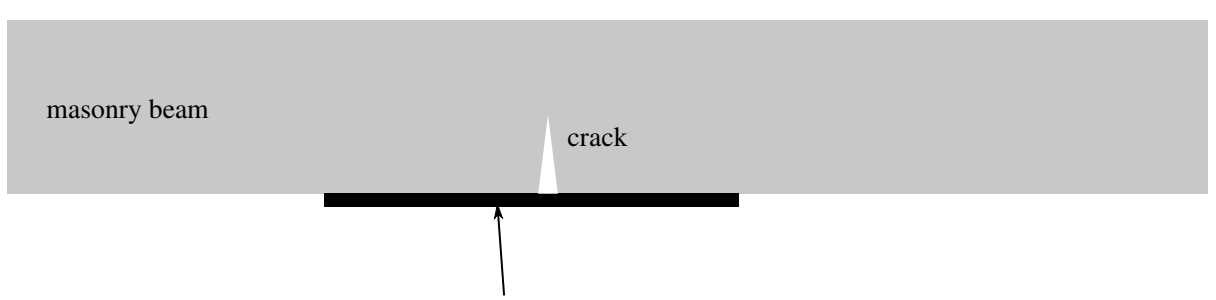

textile with embedded polymer optical fiber

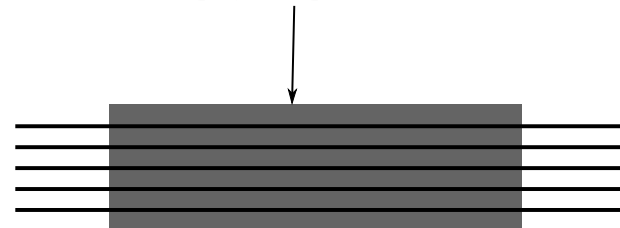

Fig. 26 Smart textile with embedded with plastic optical fiber to measure crack propagation

Seok-Ga Pagoda in Kyung Ju, Korea, was built in the 8th century in the court of the Bulkook (Bulguksa) temple. After centuries of service, the three-storey pagoda was subjected to differential settlements that caused the movement of pagoda stones that might lead to serious damage. Long-gauge SOFO interferometers were installed along the base of the pagoda to monitor the deformation during maintenance and conservation works [?].

For displacement monitoring, polymer optical fiber (POF) was embedded in textile for retrofitting the masonry structures of historic buildings (Fig. 26) [?,?]. With the high ultimate strain of POF (about 40\%), large local displacement (a few millimeters) caused by crack propagation can be monitored by OTDR. Depending on the capability of the OTDR, the spatial resolution can be as small as $10 \mathrm{~cm}$. The POF crack sensor can measure crack from $1 \mathrm{~mm}$ up to $20 \mathrm{~mm}$. In the testing of a single storey brick building on a shaking table to simulate damage under earthquake, two cracks were identified from the backscattered signal of an OTDR. The size of one of the cracks was $2 \mathrm{~mm}$ while the other was nearly invisible [?,?]. 
3.3 Geotechnical structures

Due to high uncertainties in the design parameters of geotechnical structures, it is desirable to collect in-situ data to verify the design assumptions, construction quality and actual behavior in service. As visual inspection and surface mounted sensors are inapplicable for this task, embedded fiber optic sensors have been developed for foundations, piles, anchorages, tunnels, dams, embankments as well as natural slopes.

\subsubsection{Foundations, piles and anchorages}

The defects of piles after construction are difficult to identify, as pile load test can only provide overall load capacity of pile. It is desirable to have embedded sensors to measure the internal response. Extrinsic Fabry-Perot Interferometric (EFPI) strain wave sensors with specially packaged sensor body that matched the aggregate size of the concrete mix were developed $[?, ?, ?]$. Eight sensors were embedded in a precasted driven pile at five different levels to monitor the arrival time of wave at different locations. The sensors are loosely mounted (rather than rigidly fixed) on the longitudinal steel reinforcing bars (Fig. 27) so the measurement is not affected by the steel reinforcement. All sensors survived after pile installation, dynamic pile test with high impact drop weight and static pile test. The bearing capacity of the pile was estimated by the results of the dynamic and static pile tests. The integrity of the pile and the subsoil conditions can be estimated by wave theory and the arrival time of the stress wave generated from an impact pulse at the pile head to the embedded EFPI at different locations of the pile.

The EFPI strain wave sensor is further optimized in performance and economy (Fig. 28) [?,?]. In the original EFPI sensor illustrated in Fig. 27, a sensor of standard length is packaged inside a steel cage. The measured strain is hence much lower than the concrete. To improve 


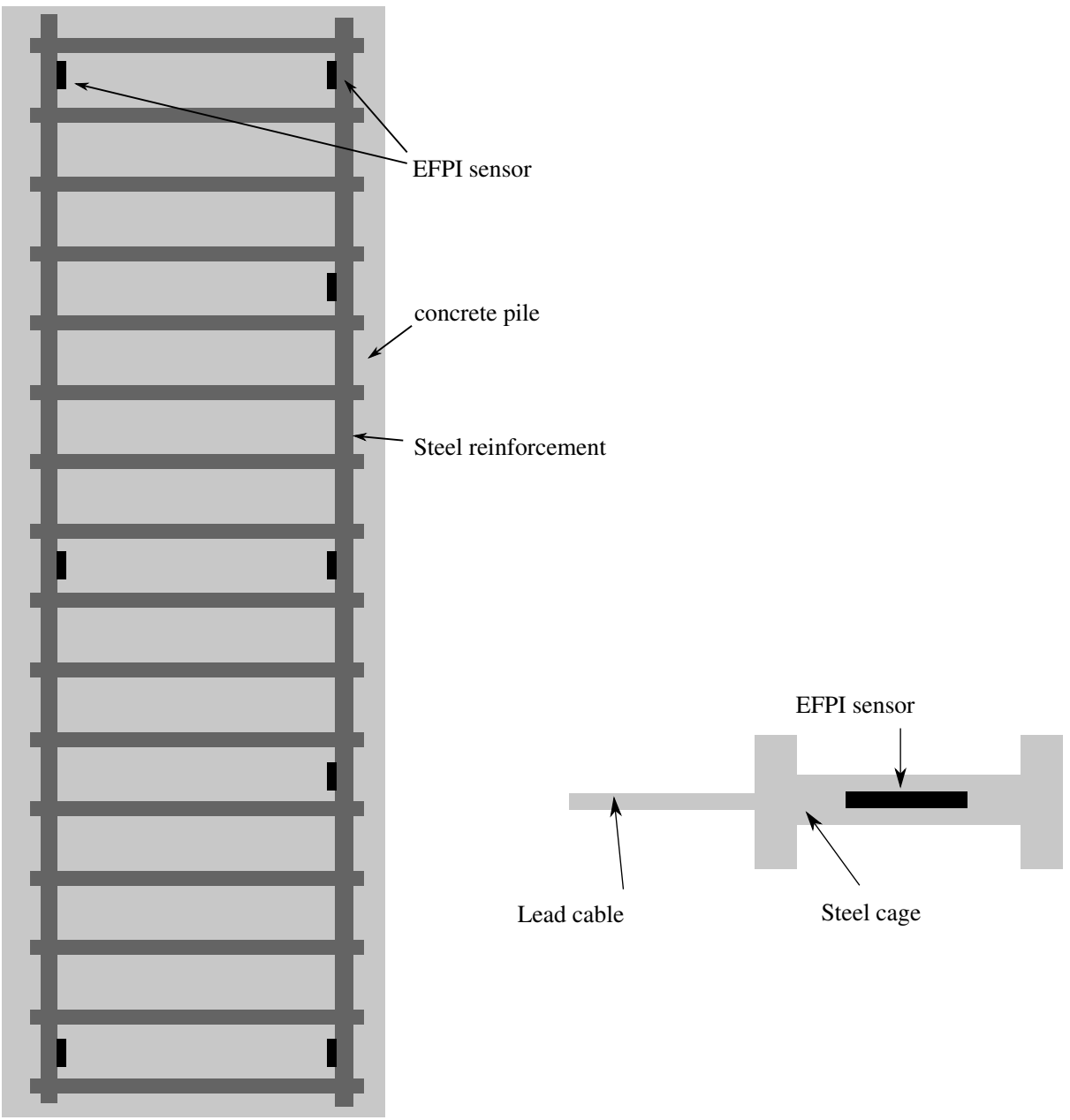

Fig. 27 Concrete pile with embedded special packaged EFPI sensors

the sensitivity, a new design is developed (Fig. 28). For this design, a gap is introduced in the steel cage. By filling the gap with soft material, the sensor exhibits very low stiffness under axial deformation. Small deformations in the concrete can therefore be easily captured. To prevent damage by bending and shear, the sensor is reinforced by small steel rods at several locations around the internal circumference. To measure the strain wave transmitting through the concrete, instead of welding the sensor on the steel reinforcement, the sensor is supported by a rod attached to the reinforcement (see Fig. 28). The sensitivity of EFPI 


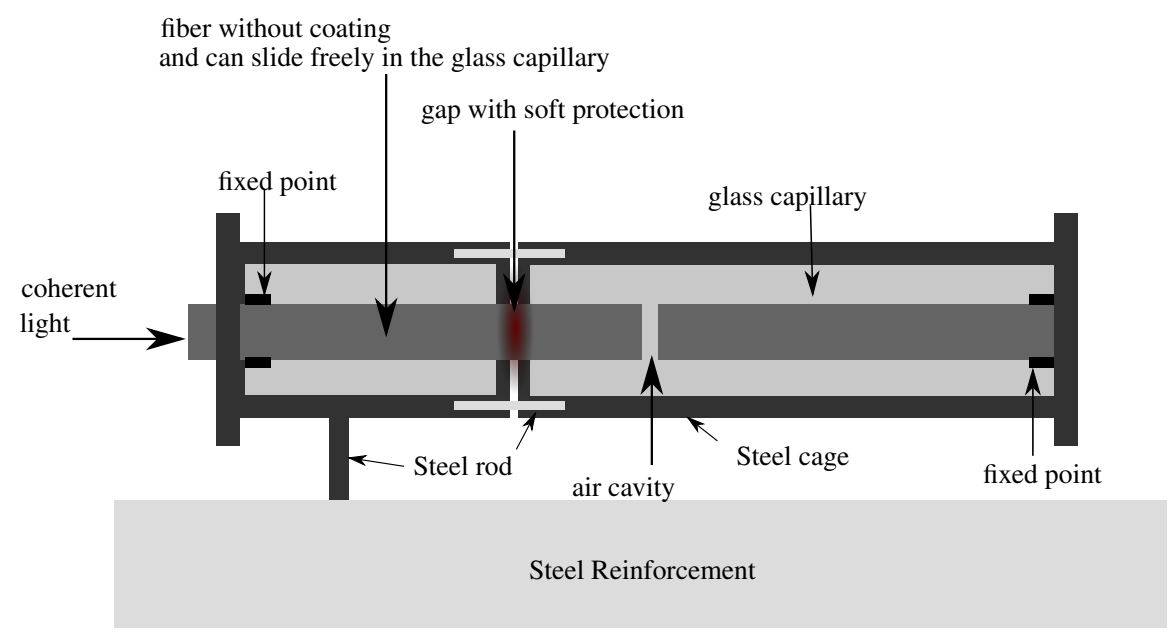

Fig. 28 Specially packaged EFPI sensors for improved performance

increases with decreasing separation between the two mirrors, but the mirrors should not touch each other at any time. The optimal mirror separation of the EFPI strain wave sensor is therefore determined by two important deformations, which are the shrinkage deformation during concrete curing and the deformation during testing and operation. Optimized EFPI strain wave sensors were tested in a large-scale cast-in-place bored model pile of $10 \mathrm{~m}$ length and $0.75 \mathrm{~m}$ diameter at the BAM Test Site in Horstwalde, Germany. The sensors were installed at three different depths at $1.5 \mathrm{~m}, 5 \mathrm{~m}$ and $8.5 \mathrm{~m}$ from the ground surface. At each level, the EFPI sensors were mounted on the reinforcement cage at $0^{\circ}, 120^{\circ}$ and $240^{\circ}$ in angular direction. In addition, a fiber Bragg grating strain sensor, a temperature sensor and a resistive strain gauge (RSG) were installed at each measurement level for comparison. The EFPI strain wave sensors detected the wave propagation from a low strain impact clearly and correlated well to the RSG signals.

The load bearing capacity of socket steel H-pile, mini-pile, soil nail and ground anchor depends mainly on the skin friction at adjacent material interface (soil or rock). Conventionally, pull-out tests are conducted on anchors with different lengths to obtain the total load 
capacity, but this cannot provide information on the skin friction distribution, which is useful for the optimization of anchorage length. The efficiency of ground anchor depends on the interfacial shear stress transfer which varies along the length of the ground anchor in a highly nonlinear manner (Fig. 29). The effective length of ground anchor, which depends on the loading and the properties of the surrounding materials, can be estimated by the distributed tensile stress along the ground anchor. To evaluate load transfer behavior, a FBG sensor array was attached on the reinforcing bar of the anchor, which was used to restrain the uplift force of the foundation plates of a sluice [?,?]. All sensors survived after the transportation from factory, where was several hundred kilometers from the site, and installation. The sensor array was then used for long-term monitoring of the anchor bonding. To make a similar smart ground anchor, several FBG strain sensors were embedded along a 7-wire steel strand [?]. FBG sensors were embedded in the central wire, while the other six wires were wrapped helically around the central one. The concept is similar to the smart cable in Fig. 12. The only difference was that the central wire consisted of a steel tube instead of FRP. The outer and inner diameters of the tube were $5.24 \mathrm{~mm}$ and $2 \mathrm{~mm}$, respectively. The FBG sensors were embedded inside the steel tube and grouted by liquid epoxy glue.

A new semi-conductor production facility in Tainan Scientific Park, Taiwan, is to be founded on a soil with poor mechanical properties. The facility requires a highly stable foundation and approximately 3000 piles are necessary. All the piles have identical mechanical properties as well as geometrical dimensions of $1.20 \mathrm{~m}$ in diameter and $35 \mathrm{~m}$ in length. SOFO sensors were mounted on the steel reinforcement of the piles before pouring of concrete to monitor the performance and behaviors of piles under push-down, pull-out and flexural full-scale on-site pile tests [?].

Two sets of piles, located at the East and West sides of the foundation respectively, were tested. Each set consisted of three piles, and each pile was tested with different loading 


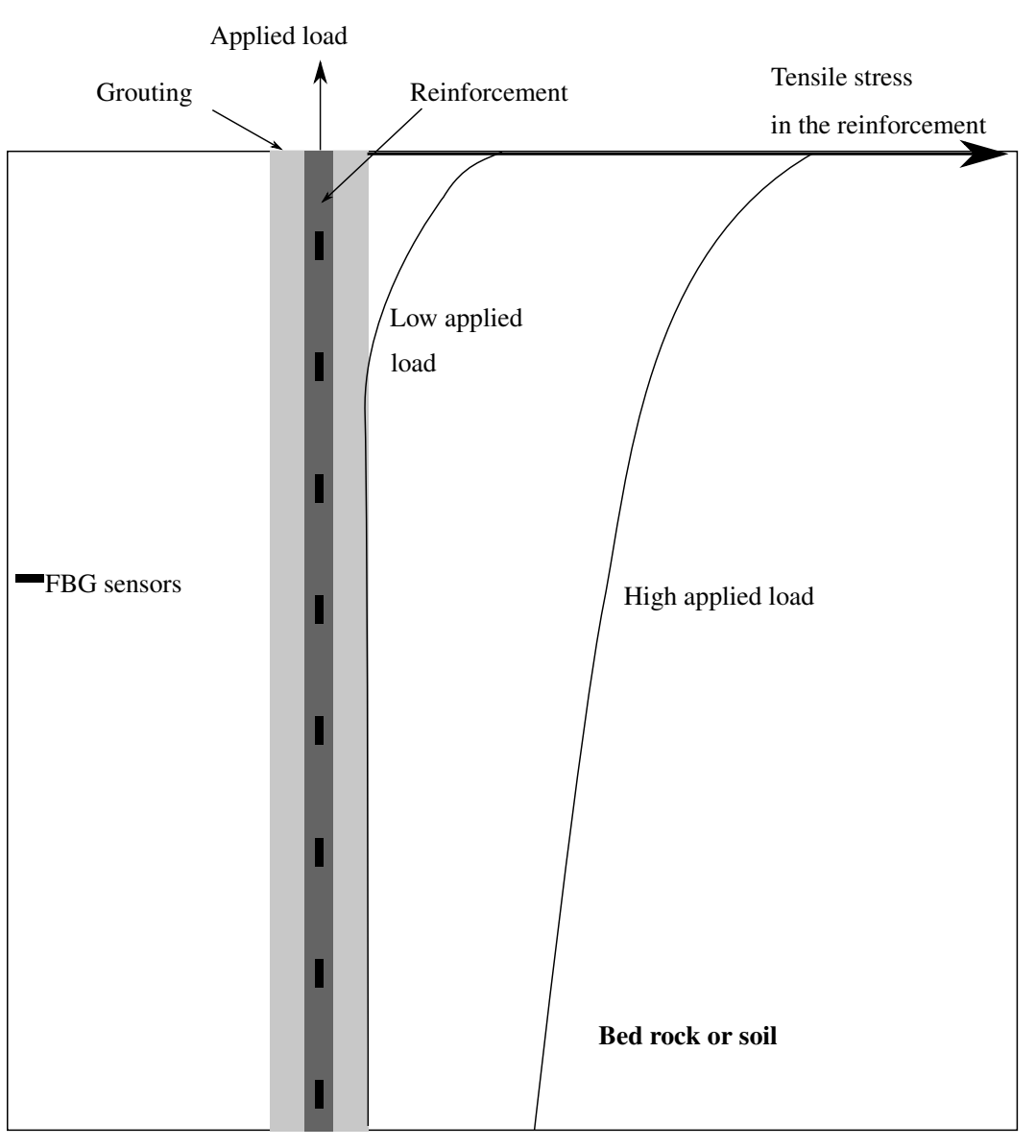

Fig. 29 Embedded FBG sensors to measure the nonlinear distribution of tensile stress along a ground anchor

types, compression, tension or lateral force, to collect information on the pile including the elastic modulus, deformation shape, skin friction distribution, lateral earth pressure along the pile, ultimate load capacity and failure mechanism. Each pile was loaded and unloaded by a hydraulic jack according to a predefined load schedule. At each pre-defined load level, the load was held unchanged for prescribed duration.

A fiber optic $\mathrm{pH}$ sensor was developed for reinforced concrete structures based on ratiometric absorption method that measured the ratio of intensities at two different wavelengths $[?, ?]$. Since relative intensity is measured, this method can overcome the instability 


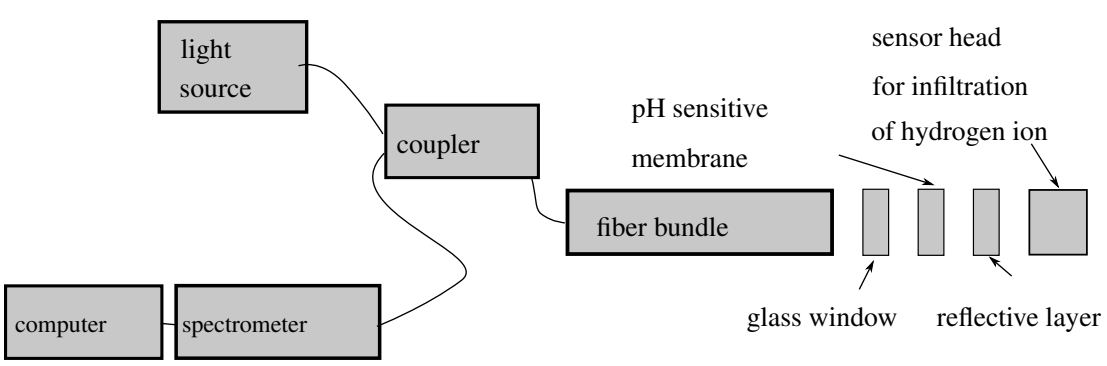

Fig. 30 Schematic diagram of the ratiometric adsorption spectrum based $\mathrm{pH}$ sensor

problems caused by decrease of the indicator concentration due to photodegradation or leaching out, drifts of the light source intensity as well as bending of optical fibers. The sensor consists of a fiber bundle, a glass window, a $\mathrm{pH}$ sensitive membrane and a reflective layer (Fig. 30). The fiber bundle maximizes the intensity of light transmission. When pH level changes, the absorbed wavelength of the $\mathrm{pH}$ sensitive membrane also changes. The absorption spectrum is measured by a spectrometer. The sensor was used to monitor steel anchor corrosion to avoid bond failure with the fixed anchorage length [?]. The sensitive range of $\mathrm{pH}$ level of the sensor is between 9.5 and 13.5. The measurement resolution is between 0.1 to $0.6 \mathrm{pH}$ at different $\mathrm{pH}$ levels. The most sensitive range is between $\mathrm{pH} 9.7$ and $\mathrm{pH}$ 11. The homogeneous sensitive membrane, prepared by colored adsorbent resin beads, exhibits long-term stability under strong basic conditions without any leaching. The response time for measuring the decrease in $\mathrm{pH}$ level is about 60 minutes, which is not a problem because the $\mathrm{pH}$ change in concrete structures is much slower than the response time. The main issue with the sensor durability is to maintain the hydrophilicity of the membrane to assure the ion diffusion and the protonation or deprotonation of the adsorbed $\mathrm{pH}$ sensitive indicator, so the membrane must stay wet during storage, application and in-service. A field trial at a harbor of Rostock in North Germany from summer 2010 demonstrated that the sensor could evaluate the alkalinity of the concrete matrix around the steel. 


\subsubsection{Tunnels}

Generally, concrete lining is installed in a tunnel for traffic safety purposes. An accurate and efficient approach to monitor the structural health of the concrete lining is highly desirable. To serve this purpose, a single mode telecommunication optical fiber was attached by epoxy adhesive on the surface of a concrete tunnel lining with inner diameter of $9.1 \mathrm{~m}$. The strain along the optical fiber was measured by Brillouin optical correlation domain analysis (BOCDA) [?,?,?]. The spatial resolution of the strain distribution is less than $100 \mathrm{~mm}$. However, the measurement accuracy might deteriorate with distance of measurement. Hence, the temperature and optical polarization issues should be considered. Theoretically, there is a trade-off between the measurable range and spatial resolution by using BOCDA. As the strain can be sampled at fast rate, the approach is also suitable for dynamic strain monitoring at any arbitrary location.

Fire hazard is a particular safety concern of tunnels. It is necessary to detect and determine the exact location of fire in a short time and take action accordingly. It is expensive and impractical to employ point sensors for fire detection. A distributed temperature sensing system based on Raman optical time domain reflectometry was installed in Xuanwu Lake tunnel in China. The length of the tunnel is $1.6 \mathrm{~km}$. A single optical fiber link was attached using fixtures with design life of 30 years along the crown of the tunnel, which is expected to be the hottest location as fire causes hot air to rise up. The installation took 4 days between 1 a.m. and 4 a.m. in the morning by a 4 -man team with a hydraulic elevation truck. The installed system was subjected to a fire test with ignition of a $50 \mathrm{~cm}$ diameter basin of diesel

(Fig. 31). The point of fire could be clearly located in 80 seconds [?]. 


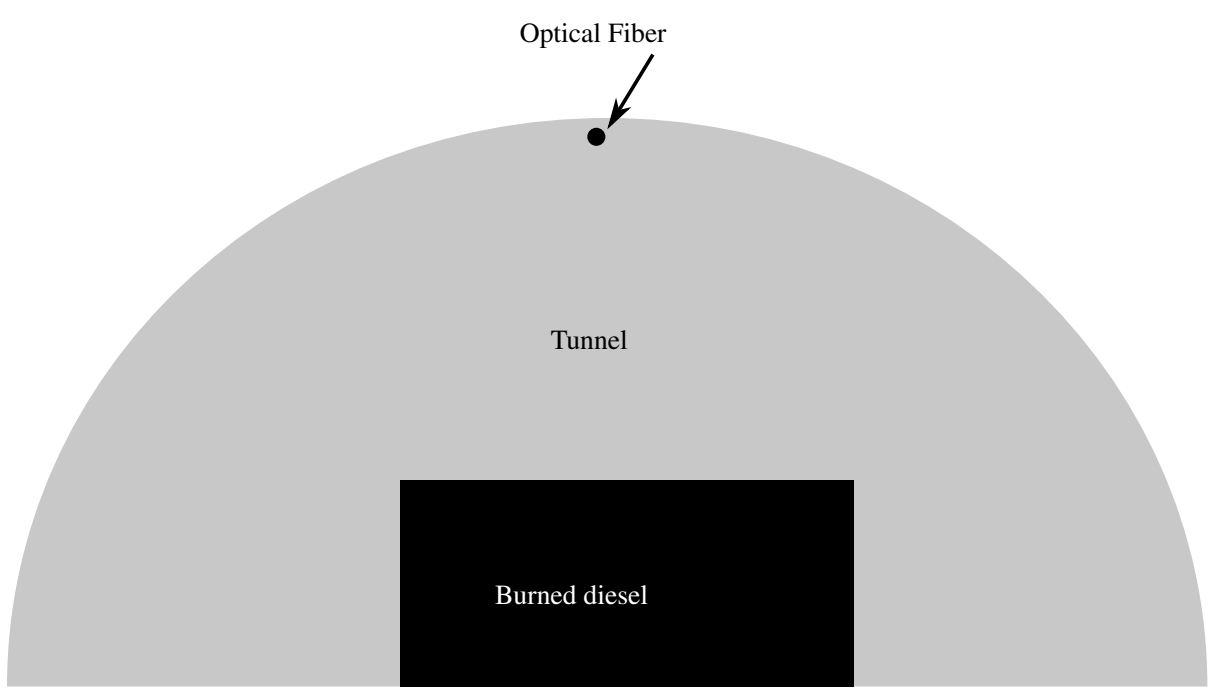

Fig. 31 Fire test of a tunnel by using Raman OTDR

\subsubsection{Dams, Embankments and Slopes}

Internal pressure of dams and embankments is an important parameter for health evaluation. With extremely high sensitivity, the interferometer is a candidate for measuring the displacement of a diaphragm under pressure. There were many pressure sensors developed based on EFPI [?] as the displacement precision of EFPI can be less than $1 \mu$ m relative to the reference point. However, when the measurement device is switched off, the reference point is lost. So, the conventional configuration of EFPI may not be suitable candidate of long term static measurement. A dual-EFPI pressure sensor that could specify a stable zero-point for every intermittent measurement was therefore developed [?,?]. One EFPI is for measurement and the other is for triggering (Fig. 32). The self-calibration procedure is started by applying compressed air to the head so that the elastic element is slightly deformed to reach a well defined reference position, which is detected by the triggering EFPI. Then, the head is bounced back to the measuring EFPI by the elastic element. The diaphragm displacement is obtained from the measuring EFPI as the displacement between the well defined reference point and 


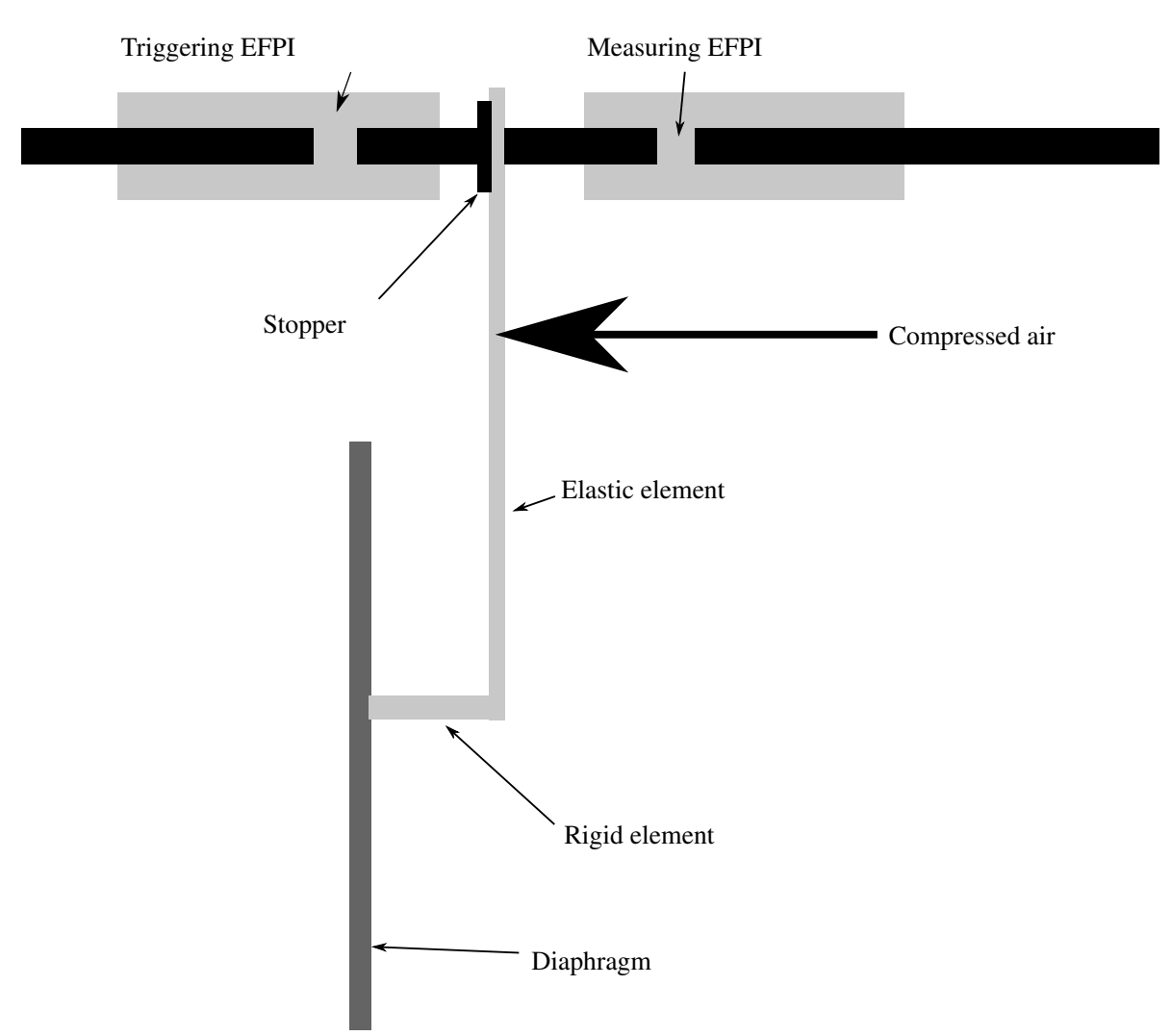

Fig. 32 Schematic diagram of self-referencing embeddable dual-EFPI pressure sensor

the position before applying the compressed air. Several of these dual-EFPI sensors have been installed at the coal mine Belchatow in Poland for long-term monitoring of the water and soil pressure

One of the early warnings of failure of retaining structures such as dams and embankment is excessive displacement. It requires a large number of point sensors to acquire adequate monitoring results, which is impractical and uneconomical. Distributed sensor provides an alternative. Geotextile is commonly used in retaining structures for reinforcement and drainage. An instrumented geotextile integrated with telecommunication silica single mode optical fiber was embedded into embankments or dikes (Fig. 33). Displacement was calculated from the distributed strain along the optical fiber obtained from stimulated Brillouin scattering 


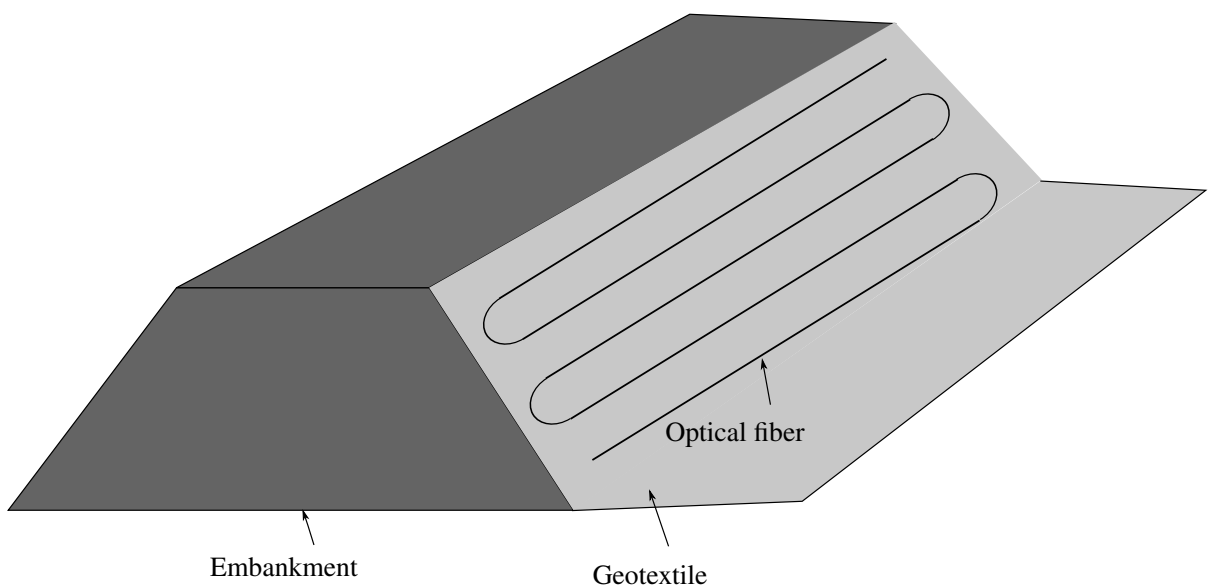

Fig. 33 Schematic diagram of instrumented geotextile for monitoring displacement of retaining structures

measured by Brillouin optical time domain reflectometry/analysis [?,?,?] or Brillouin optical frequency domain analysis (BOFDA) [?]. The cost of BOFDA is potentially lower than BOTDA. However, the ultimate strain of common silica optical fiber is only about $5 \%$, which is not sufficient for monitoring slope stability and long-term soil creep. In [?,?], the silica optical fiber was replaced by polymer optical fiber (POF) with about $40 \%$ ultimate strain. The distributed strain along the POF was measured by optical time domain reflectometer (OTDR) of Rayleigh scattering instead of stimulated Brillouin scattering. The intensity of the backscattered light was found to vary linearly with the strain of POF up to $16 \%$. To minimize the spatial blur due to the modal dispersion of the pulse from the OTDR, the use of graded POF was recommended. The instrumented geotextile with both silica and polymer optical fiber was tested in a field trial in an open brown coal pit near Belchatow, Poland. All the sensors survived after installation with heavy machineries and the maximum strain measured in the POF was more than $100 \mathrm{~mm} / \mathrm{m}$.

The State of Louisiana Office of Coastal Protection and Restoration (OCPR) plans to deploy a State-of-the-Art Intelligent Flood Protection Monitoring, Warning and Response System (IFPRMWRS) at strategic locations within the flood control systems for which it is 
responsible. The IFPRMWRS has to be able to monitor the condition of the flood protection system at all times, both during daily operation and most importantly during a rare but devastating hurricane or flood events with expected 500-year return period. As part of the project, a section of the levee was instrumented with SMARTProfile distributed optical fiber sensors based on Brillouin scattering. These sensors are used to monitor different parameters including (i) joint movements, (ii) shearing due to differential settlements, (iii) temperature changes due to leakages, seepage and overtopping.

For natural slope, it is difficult to embed instrumented geotextile to monitor the displacement profile. Instead, a fiber Bragg grating (FBG) based self temperature compensated in-place inclinometer was developed and installed at a road side slope at Luk Keng Road, Sheung Shui, Hong Kong [?,?]. Several instrumented segments are embedded in a polyvinyl chloride (PVC) casing (Fig. 34). The outer and inner diameters of the PVC casing are $70 \mathrm{~mm}$ and $60 \mathrm{~mm}$, respectively. Each instrumented segment consists of a deformable tube of about $1.5 \mathrm{~m}$ long, which is made of polycarbonate (PC), fixed at two ends by rigid stainless steel tubes. The position of the stainless steel tubes are guided by two pairs of sliding wheels, which provides constraints from the two longitudinal slots in the PVC casing. For each PC tube segment, two pairs of FBG strain sensors are fixed on the surface at two different cross sections. At each cross section, the two FBG strain sensors are glued at opposite sides of the circumference of the tube with additional epoxy on the surface for protection. The temperature induced strain can be calculated from the average of the two strain values at the same cross section. The bending strain can be obtained by subtraction the temperature induced strain. Since PC is a homogeneous and isotropic material, the curvature, which is independent to temperature, can be estimated from the bending strain at each cross section by elementary beam theory. The only loadings on the beam are the relative lateral displacement and rotation between two adjacent stainless steel tubes. From the bending strains at the two 


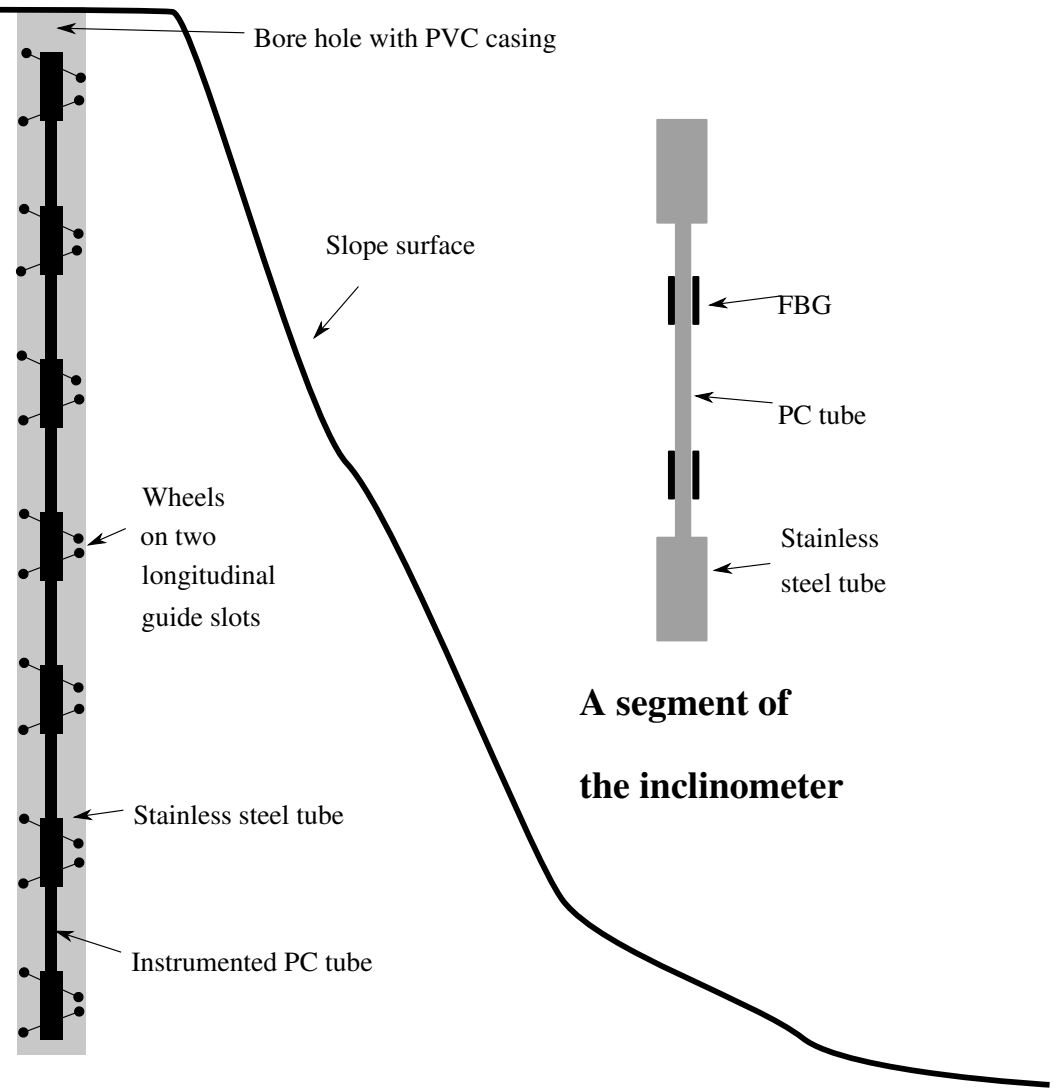

Fig. 34 Schematic diagram of a FBG based inclinometer

cross sections, the two unknown loadings can be solved and the displacement profile along the inclinometer can be computed segment by segment. The displacement profile obtained from the installed inclinometers showed that the slope movement accumulated gradually in wet season and enabled the main sliding surface in the slope to be identified. If two orthogonal lateral displacement profiles are required, four additional FBG sensors should be attached at each segment on two orthogonal planes.

The Luzzone Dam, an arch dam in Switzerland, was raised by $17 \mathrm{~m}$ in height to increase its capacity. Mass concrete was poured in-situ. Both long-gauge SOFO interferometers and Brillouin distributed temperature sensors were installed. Long-gauge SOFO interferometers 


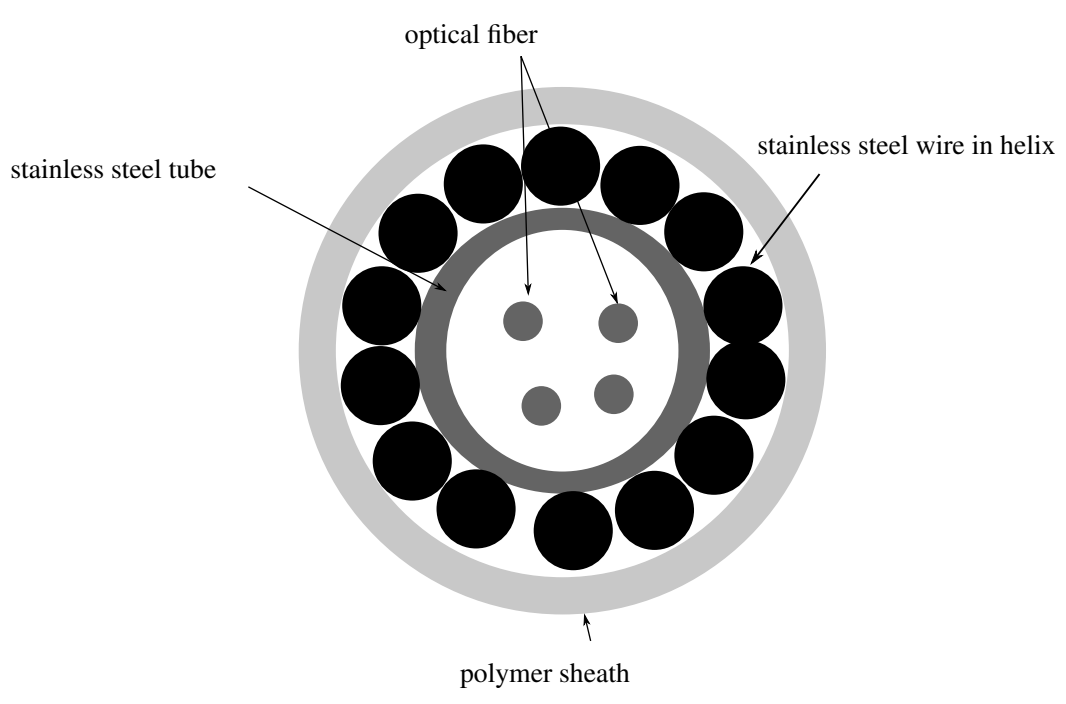

Fig. 35 Schematic diagram of the loose tube for distributed temperature measurement

were embedded into the newly casted concrete to monitor the shrinkage of the newly poured concrete and the long-term new-old concrete interaction [?,?]. All the sensors survived after concrete pouring and they could monitor the deformation of the dam over years of operation. The monitoring results were compatible with finite element simulations. The internal temperature was monitored from a Brillouin distributed fiber sensor formed by a telecommunication silica single mode optical fiber [?,?]. The sensor was installed in an undulating "serpentine" configuration before the concrete pouring. The sensor was contained in a stainless steel loose tube, protected by stainless steel armoring wires and a polymer sheath (Fig. 35). The steel tube and steel wires provided mechanical protection while the polymer sheath protected the steel from corrosion. The sensor could operate over large temperature ranges by employing appropriate coating of the optical fiber. The optical fiber was long enough to avoid any tensile strain, and hence, only thermal induced strain was measured.

Temperature sensing is not only for monitoring the heat of hydration released by newly poured mass concrete. It can also provide information on seepage through embankment 
dam and hence estimate internal erosion. Since the temperature of the water surface varies seasonally, the seepage of water through the embankment dam will cause similar temperature variation inside the dam [?]. Generally, constant temperature may be the sign of little seepage, while large temperature variations may be a sign of significant seepage. In [?], the temperature distribution along an embedded optical fiber was monitored by Raman optical time domain reflectometer. Temperature changes of $0.01^{\circ} \mathrm{C}$ could be detected, so seepage flow anomalies could be identified and evaluated with numerical analysis from the natural temperature variations observed after a few days of measurements [?].

The hydropower plant on the Daugava River in Lativa was 40 years old. The joint between two adjacent blocks of the dam was sealed by bitumen. The abrasion of the joints might redistribute the loading on the concrete blocks. The distributed strain of concrete arm next to the joint was measured by stimulated Brillouin scattering. To perform the measurements, a glass fiber reinforced polyphenylene sulfide (PPS) tape with $0.20 \mathrm{~mm}$ thickness and $13.0 \mathrm{~mm}$ width was attached on concrete surface (Fig. 36). Additional loose fiber embedded in steel tube with plastic sheath (Fig. 35) was installed in parallel to measure the distributed temperature from the stimulated Brillouin scattering for compensation of the strain measured by the sensing tape [?].

Water leakage may change the moisture content of surrounding soil. An optical fiber sensor was developed to measure the absorption spectrum of evanescent field as the existence of water molecule exhibits a unique signature of the absorption spectrum [?]. Step-index multimode low hydroxyl group plastic clad silica fiber with $600 \mu \mathrm{m}$ core diameter, $630 \mu \mathrm{m}$ cladding diameter and $1030 \mu \mathrm{m}$ buffer diameter was used. The operative wavelength was in between $1250 \mathrm{~nm}$ and $2150 \mathrm{~nm}$ in the near infrared range. The cladding of the $10 \mathrm{~cm}$ sensing portion of the optical fiber was removed by surgical blade and acetone. The bare fiber probe could measure the change in water content in dry sand and transport of pure 
polyimide optical fiber

145 microns diameter

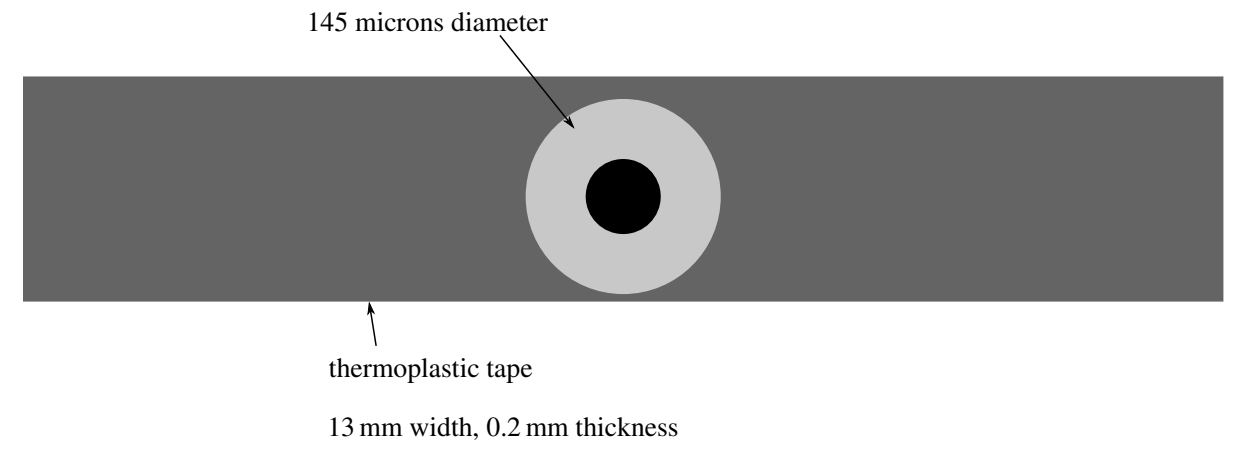

Fig. 36 Schematic diagram of surface-mounted sensor for distributed strain measurement

mineral oil in saturated sand. To detect soluble trichloroethylene, alkanes, aromatics and chlorinated hydrocarbons with the same approach, instead of bare fiber probe, cyclic olefin copolymer, which is a kind of lipophilic polymers, was coated as the new cladding and sensing part of the fiber. The laboratory results showed that the different types of organic substances can be distinguished from the signature of the absorption spectrum. However, more sophisticated technique should be used to identify the exact chemical in the same category. For example, heptane, octane, and dodecane all belong to alkanes group and they show similar characteristics of the absorption spectrum. The difference in the absorption spectrum among them is in the relative amplitude of different peaks at certain wavelengths. In reality, noise is also present, so accurate determination of amplitude ratio is difficult. With the similar sensing principle using different cladding material and $\mathrm{pH}$ sensitive chromoionophores, $\mathrm{pH}$ sensor was developed [?]. Porous cellulose acetate was used as the cladding material to carry the $\mathrm{pH}$ sensitive chromoionophores, thiazole yellow and congo red. For better light scattering and absorption of the indicator, the porosity should be high while the pore size of the cladding material should be less than half of the wavelength of transmitting light in the evanescent field. It can be achieved by immersing the cellulose acetate cladding in $0.1 \mathrm{M} \mathrm{NaOH}$ for 12 
hours. The resulting pore size is then in the range between $50 \mathrm{~nm}$ and $200 \mathrm{~nm}$. Experiments showed that the sensitive ranges of thiazole yellow and congo red were $\mathrm{pH} 10-12$ and $\mathrm{pH} 2-4$, respectively, and the sensor signal was reversible.

\subsection{Pipelines and Cables}

The main characteristic of pipelines and cables is the very long longitudinal length. The conventional leak detection method is based on the measurement of mass flow rate at certain points. With this approach, it is not possible to pinpoint the exact leak location, so slow and expensive visual inspection needs to be undertaken. It is impractical to apply point sensors for monitoring these kinds of structures. Several distributed sensors based on Brillouin optical time domain reflectometry (BOTDR) have therefore been developed. To measure the deflection of the pipeline, the tapes shown in Fig. 36 were glued along the whole length of a pipeline in Rimini of Italy at $0^{\circ}, 30^{\circ}$ and $150^{\circ}$ from the longitudinal direction $[?, ?]$. An additional optical fiber was placed inside a stainless steel loose tube (as in Fig. 35) and it was mounted along the longitudinal direction of the pipeline for temperature measurement. The strain resolution was about 20 microstrains with spatial resolution of $1.5 \mathrm{~m}$. The temperature resolution was about $1^{\circ} \mathrm{C}$ with similar spatial resolution. The distributed strain after temperature compensation can be used to monitor the deflection of the pipeline and the temperature distribution measurement can provide the temperature compensation of the total strain measured and pinpoint the location of gas leakage.

The leakage in pipeline induces local drop in temperature. For example, the leakage of ethylene may cool the pipeline locally to $-110^{\circ} \mathrm{C}$ when it is depressurized to its natural gaseous state. By using Raman optical time domain reflectometer, the exact location of sharp temperature drop can be located within meters in real time. Single mode telecommunication 

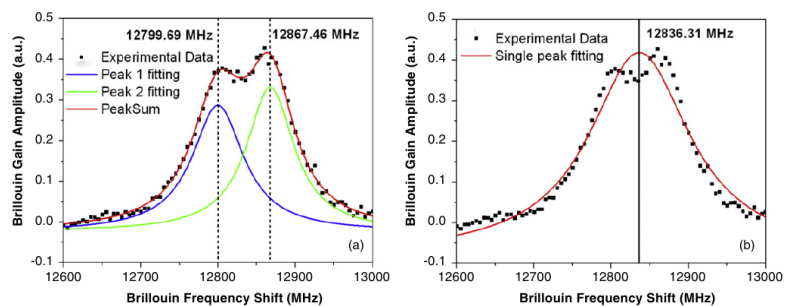

Fig. 37 Comparison between multiple-peak and single-peak fitting methods to extract the strain distribution from a Brillouin frequency spectrum

silica optical fiber can be used along the pipeline for the entire length with control stations at more than $30 \mathrm{~km}$ separation and the optical fiber can serve as telecommunication links as well as distributed temperature sensing system $[?, ?, ?]$.

Another field application example of BOTDA was the installation of an $1 \mathrm{~km}$ long telecommunication silica single mode optical fiber (embedded in FRP as in Fig. 13 ) in Daqing Oilfield to monitor the strain variation during the grouting of the downhole and perforation [?]. The FRP cable with sensing optical fiber was clamped on the surface of the pipe by stainless steel hoops and the whole pipe was covered by cement mortar to ensure deformation compatibility between the pipe and optical fiber. At the same time, FBG strain sensors were installed at critical locations such as the welded joints. Both distributed and FBG sensors survived for at least three years after installation.

The crack in a cable can be monitored by distributed Brillouin fiber sensor [?]. The stress concentration near the crack portion is usually much smaller than the spatial resolution of the Brillouin fiber sensor (about $15 \mathrm{~cm}$ ). In this case, the Brillouin frequency spectrum shows multiple peaks and the measured strain is underestimated by commonly used single peak fitting method. By using multiple-peak fitting, the maximum tensile or compressive strain is captured in about one-third of the spatial resolution $(5 \mathrm{~cm})$ as shown in Fig. 37 [?]. 
When pipelines are subjected to significant temperature change, the large thermal induced compressive stress may cause buckling. It is difficult to have prior knowledge of exact buckling location, so it is impractical to apply point sensor for monitoring. It is also impossible to identify buckling through visual inspection as the pipeline is buried underground. For this case, distributed Brillouin fiber sensor to monitor the buckling of the pipeline was demonstrated in $[?, ?, ?]$. Several telecommunication silica optical fibers were attached on the circumference of pipeline along the longitudinal direction (Fig. 38). By using the coherent probe-pump based Brillouin sensor [?], centimeter spatial resolution and 15 microstrains accuracy can be achieved over tens of kilometers. The buckling locations could be identified by the ripples of the strain distribution before the pipeline rupture. It is impractical to directly measure the hoop strain along a pipeline for several hundred kilometers. However, the distributed hoop strain can be deduced from the measured strain along the longitudinal direction of the pipeline at different angular positions at the circumference.

For ultra long submarine optical fiber cable, the exact damage location cannot be identified easily by using OTDR because the optical fibers are usually protected by external tubes and there may be certain excess length of optical fiber cable. It is a tedious task to pinpoint the cable fault location from the project documentations and then calculate based on the scale of the maps of the cable route. The accuracy of this conventional method is only around 40-50 m. By using the BOTDR, approximate fault location can be identified. Then, the cable was lifted (about $100 \mathrm{~m}$ in length) and heated up by $5^{\circ} \mathrm{C}$ locally, the distance between the heating and fault locations can be measured [?]. As shown in Fig. 39, by comparing the BOTDR reading between the preheated and heated strain distribution, the distance between the heated up point and the fault location can be estimated precisely. By using this technique, the accuracy of fault location can reach the spatial resolution of the BOTDR. 


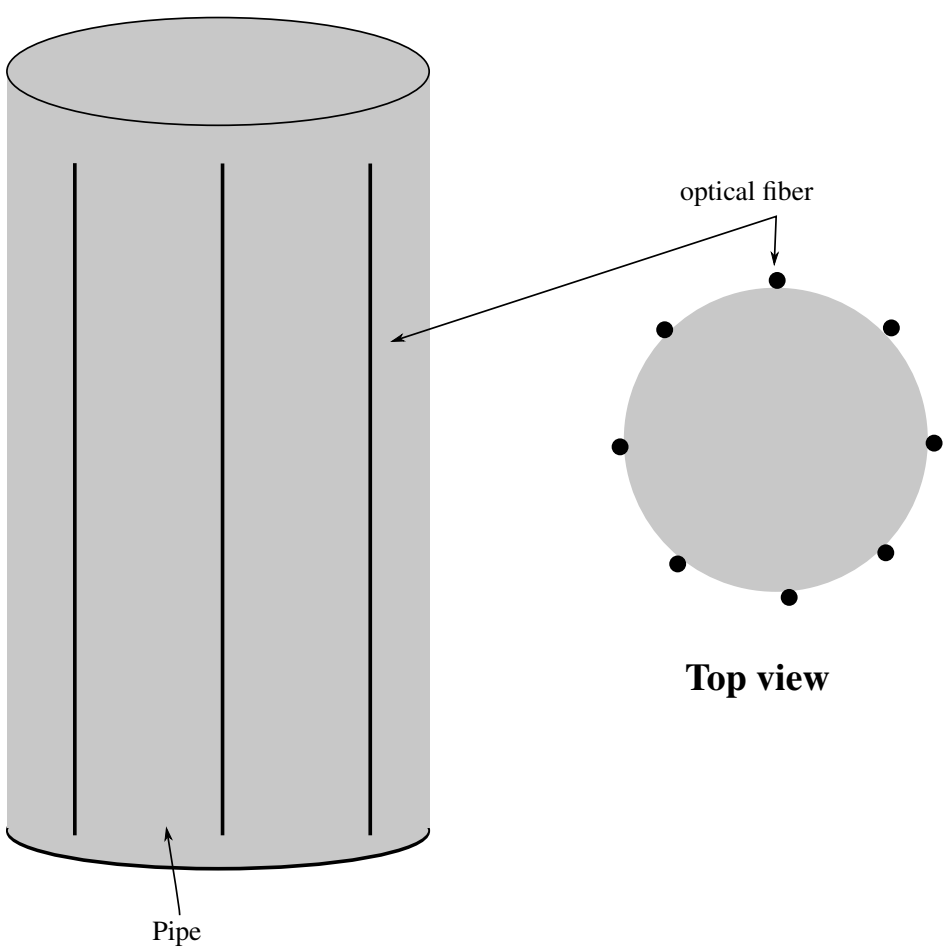

Fig. 38 Distributed sensors attached along the longitudinal direction of the pipe for buckling detection

\section{Conclusions}

In this paper, the basic principles of commonly used optical fiber sensors (OFS) are briefly reviewed, followed by description of State-of-the-Art applications of various OFS in monitoring of highway structures, building structures, geotechnical structures, pipelines as well as cables. While it is impossible to include all the available examples of OFS technologies and application techniques, the selected examples are either mature and already validated extensively on-site or in the final stage of laboratory prototyping. This paper covers only the sensor design, implementation techniques and basic sensor performance while details of the measurement results, signal processing methods and data analysis algorithms can be found in the references. 
BOTDR reading
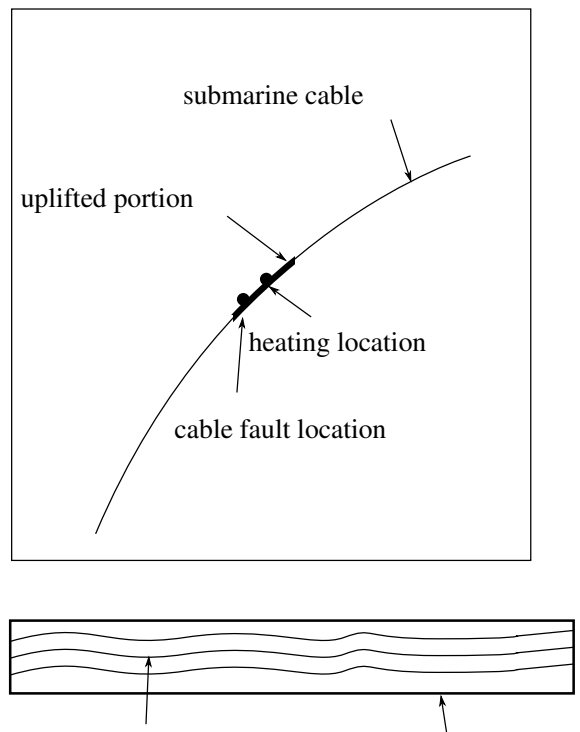

cable casing
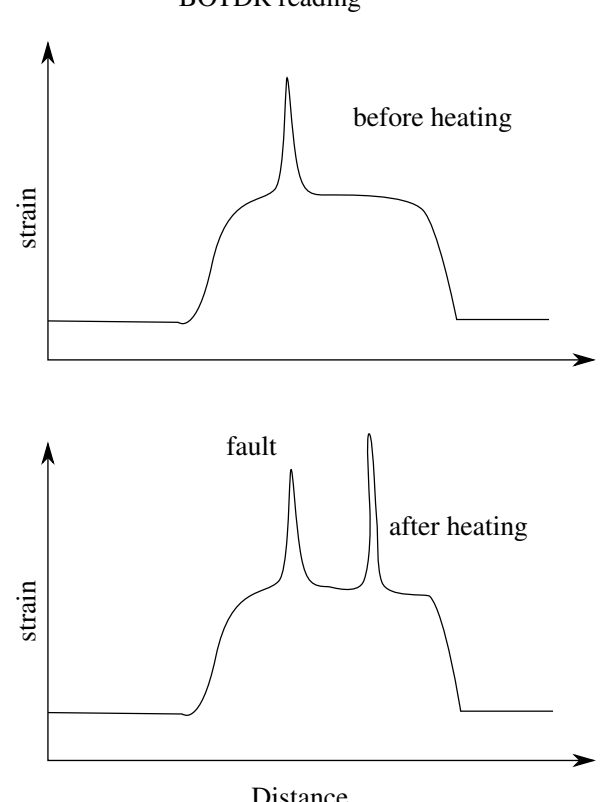

Fig. 39 Schematic diagram of cable fault locating by BOTDR

In conclusion, the development of OFS is becoming mature and they have replaced conventional sensors in various real world applications. In particular, with the capability of quasi-distributed sensing using multiplexed FBG sensors as well as distributed sensing by time domain or frequency domain reflectometry, OFS provide a technical and economical feasible solution for the monitoring of large scale structures that are commonly found in civil engineering. The various application examples presented in this paper should be useful to engineers interested in the use of OFS in their work, and can hopefully stimulate wider applications of OFS technologies in practice.

\section{References}

